\title{
Programmability of covariant quantum channels
}

\author{
Martina Gschwendtner ${ }^{1,2}$, Andreas Bluhm ${ }^{3}$, and Andreas Winter ${ }^{4,5}$ \\ ${ }^{1}$ Munich Center for Quantum Science and Technology (MCQST), 80799 München, Germany \\ ${ }^{2}$ Zentrum Mathematik, Technical University of Munich, 85748 Garching, Germany \\ ${ }^{3}$ QMATH, Department of Mathematical Sciences, \\ University of Copenhagen, 2100 Copenhagen, Denmark \\ ${ }^{4}$ Institució Catalana de Recerca i Estudis Avançats (ICREA), \\ Pg. Lluis Companys, 23, 08001 Barcelona, Spain \\ ${ }^{5}$ Grup d'Informació Quàntica, Departament de Física, \\ Universitat Autònoma de Barcelona, 08193 Bellaterra (Barcelona), Spain
}

\begin{abstract}
A programmable quantum processor uses the states of a program register to specify one element of a set of quantum channels which is applied to an input register. It is well-known that such a device is impossible with a finite-dimensional program register for any set that contains infinitely many unitary quantum channels (Nielsen and Chuang's No-Programming Theorem), meaning that a universal programmable quantum processor does not exist. The situation changes if the system has symmetries. Indeed, here we consider group-covariant channels. If the group acts irreducibly on the channel input, these channels can be implemented exactly by a programmable quantum processor with finite program dimension (via teleportation simulation, which uses the Choi-Jamiołkowski state of the channel as a program). Moreover, by leveraging the representation theory of the symmetry group action, we show how to remove redundancy in the program and prove that the resulting program register has minimum Hilbert space dimension. Furthermore, we provide upper and lower bounds on the program register dimension of a processor implementing all group-covariant channels approximately.
\end{abstract}

\section{Introduction}

Programmable quantum processors are devices which can apply desired quantum operations, specified by the user via program states, to arbitrary input states. This is convenient because one machine can be used to implement several operations and it resembles very much the way classical computers work based on classical programs and input data. Therefore, quantum processors have been studied since the early days of quantum information theory. In 1997, Nielsen and Chuang proved the No-Programming Theorem which states that it is not possible to implement infinitely many unitary channels exactly with finitedimensional program register [32], i.e. exact universal programmable quantum processors are impossible. However, exact programmable quantum processors were studied for special families of quantum operations [21] and approximate programmable quantum processors

Martina Gschwendtner: martina.gschwendtner@tum.de

Andreas Bluhm: bluhm@math.ku.dk

Andreas Winter: andreas.winter@uab.cat 
were considered in several contexts $[4,13,20,22,24,36,37,39]$. The question of optimal program dimension (the dimension of the program register) for an approximate universal quantum processor was only recently answered. The works [27] and [47] provided new upper and lower bounds on the program dimension applying methods from Banach space theory and quantum entropies, respectively. In this work, we consider a special class of channels, the covariant channels, i.e. a programmable quantum processor that implements all channels which are covariant with respect to representations $U, V$ of a compact Lie group. Note that, in particular, all results also hold for finite groups. Symmetries are of fundamental importance in physics, since they give rise to conserved quantities via Noether's theorem [33]. In open systems, these symmetries arise as covariant quantum channels and are studied using tools from quantum information theory [11, 29]. From a practical point of view, symmetries often simplify problems and break the curse of dimensionality, thus making them amenable to rigorous analysis. For these reasons, covariant quantum channels appear in many different settings, such as channel discrimination, capacities and communication tasks (see Ref. [30] and the references therein). Since this is a special set, the question arises whether those channels can be implemented exactly by a programmable quantum processor. In this article, we show that an exact implementation is possible if the group acts irreducibly on the channel input. While we prove upper bounds on the program dimension for general representations $U$, we focus on the case in which $U$ is irreducible. As any representation can be decomposed into irreducible ones, it is natural to start studying this scenario before investigating more general representations in future work.

In Section 2, we present some preliminaries and our notation. We consider exact programmability of group-covariant channels in Section 3, where we first look at a method based on extreme points in Subsection 3.1. We show that processors have a particularly simple measure-and-prepare form if and only if the commutant of the tensor representation is abelian. This yields a program dimension equal to the number of irreducible representations occurring in the direct sum decomposition of the tensor representation in Corollary 19. Subsection 3.2 discusses the structure of the commutant of the tensor representation in more detail. We give a different construction of covariant programmable quantum processors based on teleportation in Subsection 3.3. This construction is subsequently concatenated with a compression map which allows us to utilize the special structure of the Choi-Jamiołkowski states corresponding to the covariant channels. This leads to Theorem 25 , where we show that we obtain a program dimension of at most the sum of the dimensions of the blocks occurring in the structure of the Choi-Jamiołkowski states. After the analysis of exact programmability, we consider an approximate version thereof (see Section 4). First, we provide approximate upper bounds in the case of arbitrary representations $U, V$ in Proposition 29. They are in general worse than the exact bounds in Theorem 25, but they apply more generally. This result is the only one in which we consider arbitrary representations $U$ instead of irreducible ones. In Theorem 31, we provide lower bounds on the program dimension of approximate covariant quantum processors. In particular, this shows that the construction in Theorem 25 is optimal for the exact case.

\section{Preliminaries}

We use the following notation: Let $d_{1}, d_{2} \in \mathbb{N}$. We denote the set of bounded linear operators $\mathcal{H}_{1} \rightarrow \mathcal{H}_{2}$ with $d_{1}$ - and $d_{2}$-dimensional Hilbert spaces $\mathcal{H}_{1}$ and $\mathcal{H}_{2}$ by $\mathcal{B}\left(\mathcal{H}_{1}\right)$ and $\mathcal{B}\left(\mathcal{H}_{2}\right)$, respectively. The set of all $d_{1}$-dimensional density operators is

$$
\mathcal{D}\left(\mathcal{H}_{1}\right)=\left\{\rho \in \mathcal{B}\left(\mathcal{H}_{1}\right) \mid \rho \geq 0, \operatorname{tr}(\rho)=1\right\},
$$


analogously for $d_{2}$. The set of all pure states is denoted by $\mathcal{D}_{P}$. A quantum channel is a completely positive trace-preserving map $T: \mathcal{B}\left(\mathcal{H}_{1}\right) \rightarrow \mathcal{B}\left(\mathcal{H}_{2}\right)$, where $d_{1}, d_{2} \in \mathbb{N}$. We write $\operatorname{CPTP}\left(\mathcal{H}_{1}, \mathcal{H}_{2}\right)$ for this set, and $\operatorname{CPTP}(\mathcal{H})$ if $d_{1}=d_{2}=d$.

We review some basic results from representation theory which we need in our analysis. For further details we refer to Ref. [38]. Throughout the paper we shortly write compact group instead of compact Lie groups.

Definition 1 (Unitary representation). Let $G$ be a compact group. A unitary representation of $G$ is a continuous homomorphism from $G$ to the unitary operators $\mathcal{U}_{1}:=\mathcal{U}\left(\mathcal{H}_{1}\right)$ on some complex, $d_{1}$-dimensional Hilbert space $\mathcal{H}_{1}$.

Since all representations in this paper will be unitary, we will refer to them as representations for brevity. Any representation can be decomposed into irreducible representations, which are its fundamental building blocks because those cannot be decomposed any further.

Definition 2 (Irreducible representation [38, p. 157]). A unitary representation $U$ of a group $G$ on a finite-dimensional vector space $\mathcal{H}_{1}$ is called irreducible representation (irrep) if and only if the only invariant subspaces of $\left\{U_{g} \mid g \in G\right\}$ are $\{0\}$ and the whole space.

A fundamental result in representation theory states that we can decompose any unitary representation of a compact group into a direct sum of irreducible representations.

Lemma 3 (Direct sum decomposition [38, Theorem VII.9.3.]). Every representation of a compact group $G$ is equivalent to a direct sum of irreps.

Irreducible representations of abelian groups have a particularly easy form:

Corollary 4 (Degree irrep abelian group [38, Corollary II.4.3.]). If $G$ is abelian, every irrep has dimension $d=1$.

The image of the representation, i.e. the set of unitaries $U_{g}, g \in G$, generates a matrix algebra (i.e. a finite-dimensional unital $*$-algebra of linear operators). To study symmetries in the next chapter, we define the commutant of this algebra as:

Definition 5 (Commutant). Let $\mathcal{A}$ be a matrix algebra on the Hilbert space $\mathcal{H}_{d}$. Its commutant is

$$
\mathcal{A}^{\prime}:=\{B \mid B A=A B \forall A \in \mathcal{A}\} .
$$

Note that the commutant is again a matrix algebra. If the algebra has a special structure, its commutant also has a corresponding structure which directly follows from the structure of $\mathcal{A}$ and the definition of a commutant.

Lemma 6 (Structure of the commutant [38, Thm IX.11.2.]). Let $U$ be a unitary representation of a compact group $G$ on $\mathcal{H}_{1}$, which can be written as $\mathcal{H}_{1}=\underset{k=1}{\bigoplus}\left(\mathcal{H}_{k} \otimes \mathcal{H}_{k}^{\prime}\right)$ such that $U_{g}=\underset{k=1}{K} U_{g}^{(k)} \otimes \mathbb{1}_{n_{k}}$ for all $g \in G$ where $U^{(k)}, k \in\{1, \ldots, K\}$, are irreps of $G$. Furthermore, let $\mathcal{A}(U)$ be the operator algebra generated by the $\left\{U_{g}\right\}_{g \in G}$ and $\mathcal{A}^{\prime}(U)$ the corresponding commutant. Then,

$$
\begin{aligned}
\mathcal{A}(U) & =\bigoplus_{k=1}^{K} \mathcal{B}\left(\mathcal{H}_{k}\right) \otimes \mathbb{1}_{n_{k}}, \\
\mathcal{A}(U)^{\prime} & =\bigoplus_{k=1}^{K} \mathbb{1}_{b_{k}} \otimes \mathcal{B}\left(\mathcal{H}_{k}^{\prime}\right) .
\end{aligned}
$$




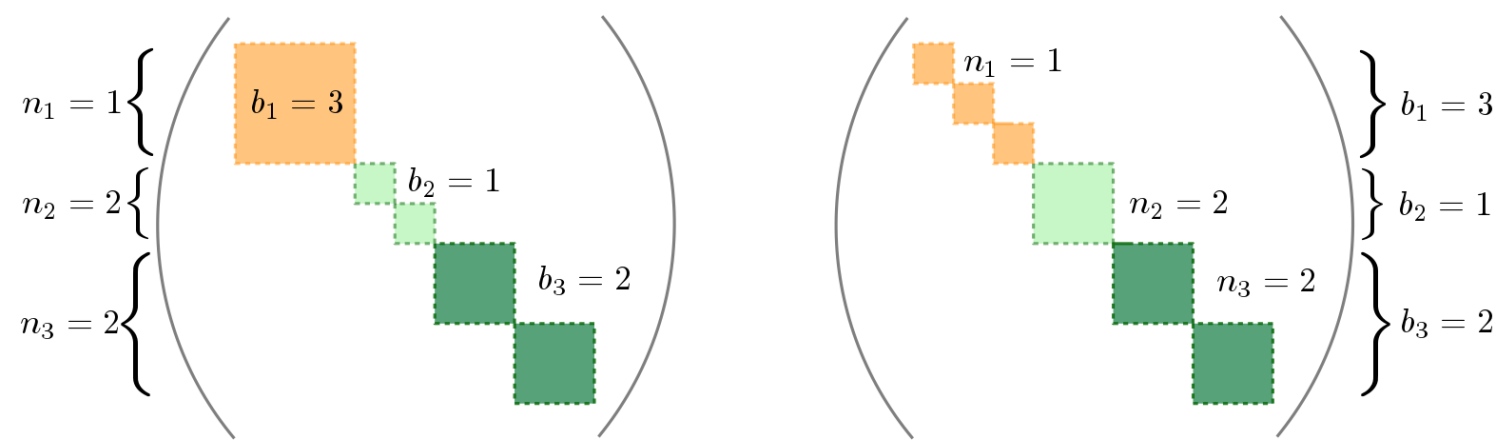

Figure 1: This figure illustrates the structure of the commutant in the case of $K=3$, i.e. 3 irreps in the direct sum decomposition.

The special structure of the commutant is illustrated in Figure 1 for a concrete example. A useful tool in the analysis of direct sum decompositions, irreps and their multiplicities is the notion of a character which is a complex number associated to each group element and related to a unitary representation through the trace operation. It is defined as:

Definition 7 (Character [38, p. 41]). Let $U: G \rightarrow G L\left(\mathcal{H}_{1}\right)$ be a representation such that $g \mapsto U_{g}$. The character $\chi: G \rightarrow \mathbb{C}$ maps every element of $G$ to a complex number according to $g \mapsto \operatorname{tr}\left(U_{g}\right)$.

In the case of a one-dimensional representation, the character is equal to the one-dimensional representation. The following theorem shows how characters can be used to study representations.

Lemma 8 (Orthogonality relations for characters [38, Theorem VII.9.5]). For all $\alpha, \beta$ irreps,

$$
\left\langle\chi_{\alpha}, \chi_{\beta}\right\rangle:=\int_{G} \overline{\chi_{\alpha}(g)} \chi_{\beta}(g) d \mu(g)=\delta_{\alpha \beta}
$$

where $\left\langle\chi_{\alpha}, \chi_{\beta}\right\rangle$ is the inner product on $L^{2}(G)=\left\{\chi:\left.G \rightarrow \mathbb{C}\left|\int_{G}\right| \chi(g)\right|^{2} d \mu(g)<\infty\right\}$ and $\mu(g)$ denotes the Haar measure on $G$.

The multiplicities of irreps in the direct sum decomposition of a representation can be calculated as follows:

Corollary 9 (Multiplicity relation [38, Corollary VII.9.6.]). Let $U$ be a representation of a compact Lie group $G$ and $\chi_{U}$ the corresponding character. Let $\chi_{\alpha}$ be the character of an irrep $\alpha$. Then,

$$
n_{\alpha}=\left\langle\chi_{\alpha}, \chi_{U}\right\rangle=\int_{G} \overline{\chi_{\alpha}(g)} \chi_{U}(g) d \mu(g)
$$

is the multiplicity of $\alpha$ in the direct sum decomposition of $U$. Note that the $n_{\alpha}$ are uniquely determined by $U$.

Definition 10 ( $U V$-covariant quantum channel). Let $G$ be a compact group and let $U$ and $V$ be representations on Hilbert spaces $\mathcal{H}_{1}$ and $\mathcal{H}_{2}$. Let $T: \mathcal{B}\left(\mathcal{H}_{1}\right) \rightarrow \mathcal{B}\left(\mathcal{H}_{2}\right)$ be a quantum channel. We call T UV-covariant if

$$
T\left(U_{g} A U_{g}^{*}\right)=V_{g} T(A) V_{g}^{*} \quad \forall A \in \mathcal{B}\left(\mathcal{H}_{1}\right), \forall g \in G .
$$

The form of such channels was studied in Ref. [30] for the case that $U$ is irreducible, $V=U$ and $\bar{U} \otimes U$ multiplicity-free. While we will allow for general $V$, most of this article is concerned with the case in which $U$ is irreducible. 
The set of all $U V$-covariant channels is represented by

$$
\mathcal{T}_{U V}:=\left\{T: \mathcal{B}\left(\mathcal{H}_{1}\right) \rightarrow \mathcal{B}\left(\mathcal{H}_{2}\right), U V \text {-covariant quantum channel }\right\} .
$$

It is standard in quantum information theory to identify quantum channels with positive matrices via the Choi-Jamiołkowski isomorphism [10, 25]. We refer to Ref. [19] for a good book on quantum information theory. Let $d_{1}=d_{2}=d$ and $|\Omega\rangle=1 / \sqrt{d} \sum_{i=1}^{d}|i\rangle \otimes|i\rangle$ be a maximally entangled state, where $\{|i\rangle\}_{i \in\{1, \ldots, d\}}$ is an orthonormal basis on $\mathcal{H}_{1}$ and $\mathcal{H}_{2}$, respectively. We define the set of all Choi-Jamiołkowski states corresponding to quantum channels $T \in \mathcal{T}_{U V}$ as

$$
\mathcal{J}_{U V}:=\left\{c_{T} \in \mathcal{B}\left(\mathcal{H}_{1} \otimes \mathcal{H}_{2}\right): c_{T}:=(\mathrm{id} \otimes T)(|\Omega\rangle\langle\Omega|) \forall T \in \mathcal{T}_{U V}\right\} .
$$

The $U V$-covariance property on channel level has a well-known correspondance on statespace level which is stated in the following lemma.

Lemma 11. The covariance property of a channel $T \in \mathcal{T}_{U V}$ w.r.t. the unitary representations $U, V$ of a group $G$ is equivalent to the condition that the corresponding ChoiJamiotkowski state $c_{T} \in \mathcal{J}_{U V}$ commutes with $\bar{U}_{g} \otimes V_{g}$, i.e. $\left[c_{T}, \bar{U}_{g} \otimes V_{g}\right]=0$ for all $g \in G$.

Proof. Using $\left(\bar{U}_{g} \otimes U_{g}\right)|\Omega\rangle\left\langle\Omega\left|\left(\bar{U}_{g} \otimes U_{g}\right)^{*}=\right| \Omega\right\rangle\langle\Omega|$, we can compute that

$$
\begin{aligned}
\left(\bar{U}_{g} \otimes V_{g}\right)^{*} c_{T}\left(\bar{U}_{g} \otimes V_{g}\right) & =\left(\bar{U}_{g} \otimes V_{g}\right)^{*}(\mathrm{id} \otimes T)(|\Omega\rangle\langle\Omega|)\left(\bar{U}_{g} \otimes V_{g}\right) \\
& =\left(\bar{U}_{g} \otimes V_{g}\right)^{*}(\mathrm{id} \otimes T)\left(\left(\bar{U}_{g} \otimes U_{g}\right)|\Omega\rangle\langle\Omega|\left(\bar{U}_{g} \otimes U_{g}\right)^{*}\right)\left(\bar{U}_{g} \otimes V_{g}\right) \\
& =c_{\tilde{T}},
\end{aligned}
$$

where $\tilde{T}(\cdot)=V_{g}^{*} T\left(U_{g} \cdot U_{g}^{*}\right) V_{g}$. Since the Choi-Jamiołkowski map $T \mapsto c_{T}$ is an isomorphism, it follows that that the $U V$-covariance property of $T$ is equivalent to the corresponding Choi-Jamiołkowski state commuting with $\bar{U}_{g} \otimes V_{g}$.

The No-Programming Theorem states that it is not possible to build a device which can implement all unitary channels, or in fact any infinite set of unitaries, exactly and with a finite-dimensional program register. In this work, we consider a setting where the NoPogramming Theorem is not applicable because we do not want our processor to implement all unitary channels but a family with a certain symmetry consisting of possibly noisy quantum operations. Therefore, we study exact programmability first before considering an approximate version.

\section{Exact and Approximate Programmability}

We consider a programmable quantum processor that implements all $U V$-covariant channels for a unitary representation $U$ of a compact group $G$.

We define a programmable quantum processor with input $\rho \in \mathcal{D}\left(\mathcal{H}_{1}\right)$ that implements all covariant channels $T \in \mathcal{T}_{U V}$ with program states $\pi_{T} \in \mathcal{D}\left(\mathcal{H}_{P}\right)$ of dimension $d_{P}$. This is schematically illustrated in Figure 2. Mathematically, we define this processor as follows:

Definition $12\left(\epsilon-\mathrm{PQP}_{\mathcal{C}}\right)$. Let $\mathcal{H}_{1}$ and $\mathcal{H}_{2}$ be separable Hilbert spaces. Then we call $\mathcal{P} \in$ $C P T P\left(\mathcal{H}_{1} \otimes \mathcal{H}_{P}, \mathcal{H}_{2}\right)$, with finite-dimensional $\mathcal{H}_{P}$, an $\epsilon$-programmable quantum processor for a set $\mathcal{C} \subset C P T P\left(\mathcal{H}_{1}, \mathcal{H}_{2}\right)$ of channels $\left(\epsilon-P Q P_{\mathcal{C}}\right)$, if for every quantum channel $T \in \mathcal{C}$ there exists a state $\pi_{T} \in \mathcal{D}\left(\mathcal{H}_{P}\right)$ such that

$$
\frac{1}{2}\left\|\mathcal{P}\left(\cdot \otimes \pi_{T}\right)-T(\cdot)\right\|_{\diamond} \leq \epsilon .
$$




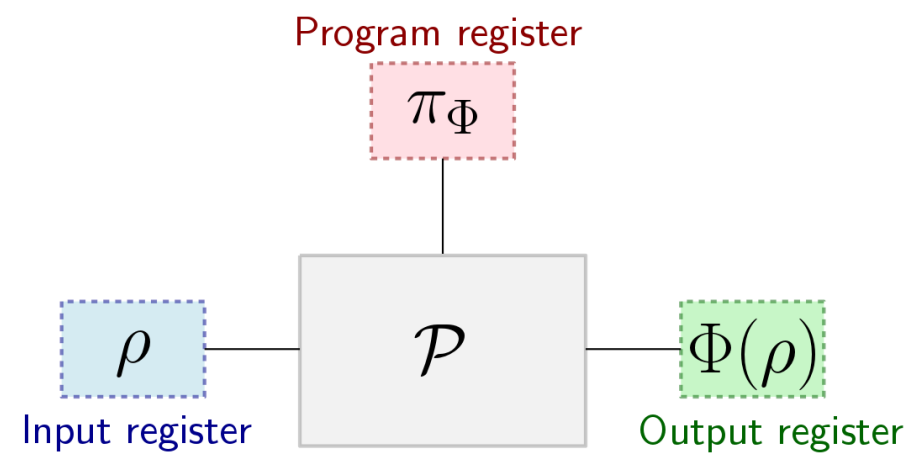

Figure 2: The figure shows a $C P Q P_{U V}$ with its input, program and output register.

To address the Hilbert spaces $\mathcal{H}_{1}, \mathcal{H}_{2}$ and $\mathcal{H}_{P}$, we refer to the former two as the input and output registers and to the latter as program register. We say that the processor $\mathcal{P}$ $\epsilon$-implements the class $\mathcal{C}$ of channels; for $\epsilon=0$ we say that $\mathcal{P}$ exactly implements the class $\mathcal{C}$, and address it as a $P Q P_{\mathcal{C}}$.

In particular, when $\mathcal{C}=\mathcal{T}_{U V}$, we write $\mathrm{CPQP}_{U V}$ for the covariant programmable quantum processor and $\epsilon-\mathrm{CPQP}_{U V}$ for the approximate version thereof. To allow for potentially mixed states in the program register is natural, since the set $\mathcal{T}_{U V}$ is convex, whereas the set of pure states is not.

Remark 13. In the literature, where the set of channels $\mathcal{C}$ often consists of isometries or even unitaries, it is customary to impose that the program state $\pi_{T}$ is pure. While this does not affect the upper bounds on the program dimension (allowing mixed states cannot make it more difficult to program a processor compared to pure states), it is in particular an essential assumption for some lower bounds when unitaries are implemented [27, 47]. On the other hand, it does not affect our lower bounds based on the properties of the Holevo information.

It is however always possible to modify a given processor $\mathcal{P} \in C P T P\left(\mathcal{H}_{1} \otimes \mathcal{H}_{P}, \mathcal{H}_{2}\right)$ to a new processor $\mathcal{P}^{\prime} \in C P T P\left(\mathcal{H}_{1} \otimes \mathcal{H}_{P}^{\prime}, \mathcal{H}_{2}\right)$ such that every channel implemented by $\mathcal{P}$, i.e. $T^{\epsilon}=\mathcal{P}\left(\cdot \otimes \pi_{T}\right)$, is implemented by $\mathcal{P}^{\prime}$ using a pure program state, i.e. $T^{\epsilon}=\mathcal{P}^{\prime}\left(\cdot \otimes\left|\psi_{T}\right\rangle\left\langle\psi_{T}\right|\right)$ for $\left|\psi_{T}\right\rangle \in \mathcal{H}_{P}^{\prime}$. The idea is to purify $\pi_{T}$ on a larger system, which gives rise to two upper bounds on $d_{P}^{\prime}=\operatorname{dim} \mathcal{H}_{P}^{\prime}$. To obtain the first, every state $\pi_{T}$ on $\mathcal{H}_{P}$ can be purified to a pure state $\left|\psi_{T}\right\rangle \in \mathcal{H}_{P} \otimes \mathcal{H}_{P}=: \mathcal{H}_{P}^{\prime}$ and we can let $\mathcal{P}^{\prime}:=\mathcal{P} \circ\left(\operatorname{id}_{\mathcal{H}_{1} \otimes \mathcal{H}_{P}} \otimes \operatorname{tr}_{\mathcal{H}_{P}}\right)$, showing that $d_{P}^{\prime} \leq d_{P}^{2}$. The second upper bound is a refinement of the first, starting from the observation that we can decompose a mixed program state into a convex combination of pure states, $\pi_{T}=\sum_{i} p_{i}\left|\psi_{T_{i}}\right\rangle\left\langle\psi_{T_{i}}\right|$, where each $\left|\psi_{T_{i}}\right\rangle \psi_{T_{i}} \mid$ implements a channel $T_{i}=\mathcal{P}\left(\cdot \otimes\left|\psi_{T}\right\rangle\left\langle\psi_{T}\right|\right)$, and hence $T^{\epsilon}=\sum_{i} p_{i} T_{i}$ is a convex combination of channels. The quantum channels $\operatorname{CPTP}\left(\mathcal{H}_{1}, \mathcal{H}_{2}\right)$ form a convex set in a space of dimension $d_{1}^{2}\left(d_{2}^{2}-1\right)$, which can be seen from the Choi-Jamiotkowski isomorphism, hence by Carathéodory's theorem $T$ can be written as a convex combination of not more than $D:=d_{1}^{2}\left(d_{2}^{2}-1\right)+1$ of the $T_{i}$, i.e. $T^{\epsilon}=\sum_{j=1}^{D} q_{j} T_{i_{j}}$, with $q_{j} \geq 0$ summing to 1 . Thus, the state $\pi_{T}^{\prime}:=\sum_{j=1}^{D} q_{j}\left|\psi_{T_{i}}\right\rangle\left\langle\psi_{T_{i}}\right|$, which has rank $\leq r:=\min \left\{D, d_{P}\right\}$, implements $T^{\epsilon}$ as well: $T^{\epsilon}=\mathcal{P}\left(\cdot \otimes \pi_{T}^{\prime}\right)$. But now we only need an r-dimensional Hilbert space $\mathcal{H}_{R}$ to purify $\pi_{T}^{\prime}=\operatorname{tr}_{\mathcal{H}_{R}}\left|\psi_{T}\right\rangle\left\langle\psi_{T}|,| \psi_{T}\right\rangle \in \mathcal{H}_{P} \otimes \mathcal{H}_{R}$. As before, we can let the processor be $\mathcal{P}^{\prime}:=\mathcal{P} \circ\left(\operatorname{id}_{\mathcal{H}_{1} \otimes \mathcal{H}_{P}} \otimes \operatorname{tr}_{\mathcal{H}_{R}}\right)$, showing $d_{P}^{\prime} \leq d_{P} \min \left\{D, d_{P}\right\}$. We conclude by noting that the first method is good if $d_{P}$ is already small, whereas the second is preferred if $d_{P}$ is large. The former is the case if we consider unitarily covariant channels (see Example 20). The latter is the case if $\mathcal{C}=\mathcal{T}_{U V}$ and the symmetries we consider are trivial, as $d_{P}$ is exponentially large in the dimension $d=d_{1}=d_{2}$ [27, 47]. 
We start with the observation that the processor can without loss of generality be chosen to be $\left(U \otimes \mathbb{1}_{d_{P}}\right) V$-covariant as well:

Proposition 14. Let $\mathcal{P}$ be an $\epsilon-C P Q P_{U V}$. Then, there exists an $\epsilon-C P Q P_{U V} \mathcal{P}^{\prime}$ with the same program dimension $d_{P}$ that is $\left(U \otimes \mathbb{1}_{d_{P}}\right) V$-covariant.

Proof. We can construct the desired processor via twirling, i.e.

$$
\mathcal{P}^{\prime}(A)=\int_{G} V_{g}^{*} \mathcal{P}\left[\left(U_{g} \otimes \mathbb{1}_{d_{P}}\right) A\left(U_{g}^{*} \otimes \mathbb{1}_{d_{P}}\right)\right] V_{g} d \mu(g) \quad \forall A \in \mathcal{B}\left(\mathcal{H}_{1} \otimes \mathcal{H}_{P}\right),
$$

where $\mu$ is the Haar measure on $G$. We compute

$$
\mathcal{P}^{\prime}\left(\rho \otimes \pi_{T}\right)=\int_{G} V_{g}^{*} T^{\epsilon}\left(U_{g} \rho U_{g}^{*}\right) V_{g} d \mu(g)
$$

for $T \in \mathcal{T}_{U V}$ and $T^{\epsilon}$ a quantum channel such that $\frac{1}{2}\left\|T-T^{\epsilon}\right\|_{\diamond} \leq \epsilon$, since $\mathcal{P}$ is an $\epsilon$-CPQP ${ }_{U V}$. Since

$$
\int_{G} V_{g}^{*} T\left(U_{g} \rho U_{g}^{*}\right) V_{g} d \mu(g)=T(\rho)
$$

for $T \in \mathcal{T}_{U V}$ by covariance, it holds that

$$
\frac{1}{2}\left\|\mathcal{P}^{\prime}\left(\cdot \otimes \pi_{T}\right)-T\right\|_{\diamond} \leq \epsilon
$$

as well. This shows that $\mathcal{P}^{\prime}$ is also an $\epsilon-\mathrm{CPQP}_{U V}$ with the same program dimension. Using the invariance of the Haar measure, it can be verified that for any $g^{\prime} \in G$,

$$
V_{g^{\prime}} \mathcal{P}^{\prime}(A) V_{g^{\prime}}^{*}=\mathcal{P}^{\prime}\left[\left(U_{g^{\prime}} \otimes \mathbb{1}_{d_{P}}\right) A\left(U_{g^{\prime}}^{*} \otimes \mathbb{1}_{d_{P}}\right)\right] \quad \forall A \in \mathcal{B}\left(\mathcal{H}_{1} \otimes \mathcal{H}_{P}\right),
$$

which shows that $\mathcal{P}^{\prime}$ is $\left(U \otimes \mathbb{1}_{d_{P}}\right) V$-covariant as desired.

Recall Lemma 11 which states that $T \in \mathcal{T}_{U V}$ is equivalent to $\left[c_{T}, \bar{U}_{g} \otimes V_{g}\right]=0$ for all $g \in G$. Due to this correspondence, we consider representations of the form $\bar{U} \otimes V$ (which are isomorphic to the adjoint representation of $G$ if $V=U$ ) with $U_{g} \in \mathcal{U}_{1}, g \in G$ and the commutant

$$
\begin{aligned}
\mathcal{K}:=\mathcal{A}(\bar{U} \otimes V)^{\prime} & =\left\{X \in \mathcal{B}\left(\mathcal{H}_{1} \otimes \mathcal{H}_{2}\right) \mid\left[X, \bar{U}_{g} \otimes V_{g}\right]=0 \forall g \in G\right\} \\
& =\bigoplus_{k=1}^{K} \mathbb{1}_{b_{k}} \otimes \mathcal{B}\left(\mathcal{H}_{k}^{\prime}\right) .
\end{aligned}
$$

Note that since $\mathcal{H}_{1}$ and $\mathcal{H}_{2}$ are finite dimensional, only $K \leq d_{1} d_{2}$ irreps can appear in the commutant with multiplicity $n_{k}>0$, where $n_{k}=\operatorname{dim}\left(\mathcal{H}_{k}^{\prime}\right)$. We identify these elements with an index $k \in\{1, \ldots, K\}$ motivated by the fact that we want to relate the irreps occuring in the direct sum decomposition of $\bar{U} \otimes V$ with the number of extreme points of $\mathcal{J}_{U V}$, for instance.

While it is not true in general that all states in $\mathcal{K}$ are Choi-Jamiołkowski states of a quantum channel, this is true if $U$ is an irrep. This is proven in the following lemma which aligns with results in Refs. [15, p. 6] and [1, p. 7].

Lemma 15. Let $\mathcal{K}$ be as defined above and let $U$ be an irrep of a compact group $G$ on $\mathcal{H}_{1}$. Let $V$ be a representation of $G$ on $\mathcal{H}_{2}$. Then $\mathcal{K} \cap \mathcal{D}\left(\mathcal{H}_{1} \otimes \mathcal{H}_{2}\right)=\mathcal{J}_{U V}$. Moreover, if $V$ is an irrep, any $T \in \mathcal{T}_{U V}$ is unital. 
Proof. "卫" Let $c_{T} \in \mathcal{J}_{U V}$. Since all elements of $\mathcal{J}_{U V}$ are Choi-Jamiołkowski states corresponding to $U V$-covariant channels, they satisfy $c_{T} \geq 0$ and $\operatorname{tr}\left(c_{T}\right)=1$ by definition. Hence, $c_{T} \in \mathcal{D}\left(\mathcal{H}_{1} \otimes \mathcal{H}_{2}\right)$. According to Lemma 11, $T \in \mathcal{T}_{U V}$ corresponds to $\left[c_{T}, \bar{U}_{g} \otimes V_{g}\right]=0$ for all $g \in G$ and thus, $c_{T} \in \mathcal{K} \cap \mathcal{D}\left(\mathcal{H}_{1} \otimes \mathcal{H}_{2}\right)$.

" $\subseteq$ " Let us refer to $\mathcal{H}_{1}$ as system $A$ and to $\mathcal{H}_{2}$ as system $B$. If we intersect $\mathcal{K}$ with the set of states $\mathcal{D}\left(\mathcal{H}_{1} \otimes \mathcal{H}_{2}\right)$, then every $\rho_{A B} \in \mathcal{K} \cap \mathcal{D}\left(\mathcal{H}_{1} \otimes \mathcal{H}_{2}\right)$ satisfies $\operatorname{tr}\left(\rho_{A B}\right)=1$ and $\rho_{A B} \geq 0$ as well as $\left[\rho_{A B}, \bar{U}_{g} \otimes V_{g}\right]=0$ for all $g \in G$. To obtain $\rho_{A B} \in \mathcal{J}_{U V}$, we additionally have to show the required property $\operatorname{tr}_{B}\left(\rho_{A B}\right)=\frac{\mathbb{1}_{d_{1}}}{d_{1}}$. Using $\left[\rho_{A B}, \bar{U}_{g} \otimes V_{g}\right]=0$, we get

$$
\operatorname{tr}_{B}\left(\rho_{A B}\right)=\operatorname{tr}_{B}\left(\left(\bar{U}_{g} \otimes V_{g}\right) \rho_{A B}\left(\bar{U}_{g} \otimes V_{g}\right)^{*}\right)=\bar{U}_{g} \operatorname{tr}_{B}\left(\rho_{A B}\right) \bar{U}_{g}^{*} .
$$

for any $g \in G$ which is equal to

$$
\operatorname{tr}_{B}\left(\rho_{A B}\right) \bar{U}_{g}=\bar{U}_{g} \operatorname{tr}_{B}\left(\rho_{A B}\right) .
$$

Since $U$ is an irrep if and only if $\bar{U}$ is, we infer due to Schur's Lemma:

$$
\operatorname{tr}_{B}\left(\rho_{A B}\right)=\lambda \cdot \mathbb{1}_{d_{1}} \quad \text { for some } \lambda \in \mathbb{C} .
$$

Taking the trace on both sides results in

$$
1=\operatorname{tr}\left(\operatorname{tr}_{B}\left(\rho_{A B}\right)\right)=\lambda \cdot \operatorname{tr}\left(\mathbb{1}_{d_{1}}\right)=\lambda \cdot d_{1}
$$

Hence, $\lambda=1 / d_{1}$. This yields $\operatorname{tr}_{B}\left(\rho_{A B}\right)=\frac{\mathbb{1}_{d_{1}}}{d_{1}}$ which we aimed to show. With the same reasoning, we can also conclude that if $V$ is an irrep,

$$
\operatorname{tr}_{A}\left(c_{T}\right)=\frac{\mathbb{1}_{d_{2}}}{d_{2}}
$$

for all $c_{T} \in \mathcal{J}_{U V}$, which implies that $T$ is unital.

In Ref. [21], the authors derived that channels implemented by a processor that is covariant with respect to the special unitary group $S U\left(\mathcal{H}_{1}\right)$ are unital using a similar argument. We will now consider how to construct covariant programmable quantum processors in the case where $\mathcal{K}$ is abelian.

\subsection{Covariant programmable quantum processors from extreme points}

In this section, we consider a special class of $\mathrm{CPQP}_{U V}$, the measure-and-prepare $\mathrm{CPQP}_{U V}$. We will show that $\mathcal{T}_{U V}$ can be implemented by a measure-and-prepare $\mathrm{CPQP}_{U V}$ if and only if the commutant $\mathcal{K}$ is abelian. Before we state the main result, we present a lemma required for its proof which is a correspondence between the commutant and the affiliated state space which we require in order to show our first main result for exact programmability.

Lemma 16. Let $U$ be an irrep of a compact group $G$, and let $V$ be another representation of $G$. Then, the following are equivalent:

i) $\mathcal{K}$ is abelian.

ii) $\mathcal{J}_{U V}$ is isomorphic to a simplex.

iii) $\mathcal{J}_{U V}$ is isomorphic to a polytope. 
Proof. "i) $\Rightarrow$ ii)" This statement is mentioned in Ref. [40] without proof. Let $B \in \mathcal{K}$ and let $\mathcal{K}$ be an abelian matrix algebra. This implies that $n_{k}=1$ for all $k \in\{1, \ldots, K\}$. Furthermore, let $K$ be the number of irreps appearing in the direct sum decomposition of $\bar{U} \otimes V$. We obtain

$$
B=\bigoplus_{k=1}^{K} \mathbb{1}_{b_{k}} \otimes x_{k}
$$

where $x_{k} \in \mathbb{C}$. Moreover, $B \geq 0$ if and only if $x_{k} \geq 0$ for all $k \in\{1, \ldots, K\}$ and $\operatorname{tr}(B)=1$ if and only if $\sum_{k} b_{k} x_{k}=1$. According to Lemma 15, extreme points of $\mathcal{K} \cap \mathcal{D}\left(\mathcal{H}_{1} \otimes \mathcal{H}_{2}\right)$ are also extreme points of $\mathcal{J}_{U V}$. The extreme points of $\mathcal{K} \cap \mathcal{D}\left(\mathcal{H}_{1} \otimes \mathcal{H}_{2}\right)$ are of the form $x_{i}=1 / b_{i}$ for some $i \in\{1, \ldots, K\}$ and $x_{l}=0$ for all $l \neq i$. We identify the $K$ extreme points of $\mathcal{J}_{U V}$ with $K$ points in $\mathbb{R}^{K}$. Therefore, $\mathcal{J}_{U V}$ is isomorphic to a $(K-1)$-simplex. "iii) $\Rightarrow$ i)" We prove this statement by contraposition, i.e. we show if $\mathcal{K}$ is non-abelian, $\mathcal{J}_{U V}$ is not isomorphic to a polytope. If $\mathcal{K}$ is non-abelian, there is a $k \in\{1, \ldots, K\}$ such that the corresponding block is of dimension $n_{k}>1$. Let us consider elements of the form

$$
B_{\varphi}=\left(\frac{1}{b_{k}}\left|\varphi_{k}\right\rangle\left\langle\varphi_{k}\right| \otimes \mathbb{1}_{b_{k}}\right) \oplus \mathbf{0},
$$

where $\left|\varphi_{k}\right\rangle\left\langle\varphi_{k}\right| \in \mathcal{D}\left(\mathcal{H}_{k}^{\prime}\right)$. The normalization $\frac{1}{b_{k}}$ yields $\operatorname{tr}\left(B_{\varphi}\right)=1$ and furthermore, $B_{\varphi} \geq$ 0, i.e. $B_{\varphi} \in \mathcal{D}\left(\mathcal{H}_{1} \otimes \mathcal{H}_{2}\right)$. These elements are extreme points of $\mathcal{J}_{U V}$ by Lemma 15 . Thus, there are infinitely many extreme points of $\mathcal{J}_{U V}$. Hence, the set cannot be isomorphic to a polytope which has finitely many extreme points by definition.

The last implication "ii) $\Rightarrow$ iii)" is obvious.

Let us now state the definition of the class of processors we consider in this section.

Definition 17 (Measure-and-prepare $P Q P_{\mathcal{C}}$ ). Let $\mathcal{H}_{1}$ and $\mathcal{H}_{2}$ be separable Hilbert spaces. Then, we call a $P Q P_{\mathcal{C}} \mathcal{P}$ with finite-dimensional $\mathcal{H}_{P}$ a measure-and-prepare $P Q P_{\mathcal{C}}$ for a set of quantum channels $\mathcal{C}$ if there exists a $K \in \mathbb{N}$, a POVM $\left\{E_{i}\right\}_{i \in\{1, \ldots, K\}} \subset \mathcal{D}\left(\mathcal{H}_{P}\right)$ and a set of quantum channels $T_{k} \in \mathcal{C}, k \in\{1, \ldots, K\}$, such that

$$
\mathcal{P}(A \otimes B)=\sum_{k=1}^{K} \operatorname{tr}\left[E_{k} B\right] T_{k}(A) \quad \forall A \in \mathcal{B}\left(\mathcal{H}_{1}\right), B \in \mathcal{B}\left(\mathcal{H}_{P}\right) .
$$

Proposition 18. Let $\mathcal{C} \subset \operatorname{CPTP}\left(\mathcal{H}_{1}, \mathcal{H}_{2}\right)$ be a convex set of quantum channels. Then, there is a measure-and-prepare $P Q P_{\mathcal{C}}$ if and only if $\mathcal{J}_{\mathcal{C}}$, the corresponding set of ChoiJamiotkowski states, is isomorphic to a polytope. Moreover, if such a measure-and-prepare $P Q P_{\mathcal{C}}$ exists, $d_{P}$ can be chosen to be the number of extreme points and the program states can be chosen pure.

Proof. We fix a quantum channel $T \in \mathcal{C}$ with its corresponding Choi-Jamiołkowski state $c_{T} \in \mathcal{J}_{\mathcal{C}}$. Since $\mathcal{J}_{\mathcal{C}}$ is isomorphic to a polytope, it is spanned by $K$ extreme points $c_{T_{k}}$ and can therefore be written as convex combination of these

$$
c_{T}=\sum_{k=1}^{K} x_{k} c_{T_{k}}
$$

where $x_{k} \in[0,1]$ and $\sum_{k=1}^{K} x_{k}=1$. By the Choi-Jamiołkowski isomorphism, there is a channel $T_{k} \in \mathcal{T}_{U V}$ corresponding to each of the extreme points $c_{T_{k}}$ of $\mathcal{J}_{\mathcal{C}}$, i.e. $T$ can be linearly decomposed

$$
T(\cdot)=\sum_{k=1}^{K} x_{k} T_{k}(\cdot)
$$


with extreme points $T_{k}(\cdot)$. We encode the $\left\{x_{k}\right\}_{k \in\{1, \ldots, K\}}$ in the program state as follows

$$
\left|\psi_{T}\right\rangle=\sum_{k=1}^{K} \sqrt{x_{k}}|k\rangle \in \mathcal{D}_{P}\left(\mathcal{H}_{P}\right),
$$

with an arbitrary orthonormal basis $\{|k\rangle\}_{k \in\{1, \ldots, K\}}$ on $\mathcal{H}_{P}$. The following processor implements $T \in \mathcal{C}$ exactly with a program register of dimension $d_{P}=K$ :

$$
\mathcal{P}(A \otimes B)=\operatorname{tr}_{\mathcal{H}_{P}}\left[\sum_{k=1}^{K} T_{k}(A) \otimes\langle k|B| k\rangle|k\rangle\langle k|\right] \quad \forall A \in \mathcal{B}\left(\mathcal{H}_{1}\right), \forall B \in \mathcal{B}\left(\mathcal{H}_{P}\right)
$$

and extended by linearity. We verify that this is indeed a measure-and-prepare $\mathrm{PQP}_{\mathcal{C}}$ :

$$
\mathcal{P}\left(\rho \otimes\left|\psi_{T}\right\rangle\left\langle\psi_{T}\right|\right)=\sum_{k=1}^{K} T_{k}(\rho) \operatorname{tr}\left[E_{k}\left|\psi_{T}\right\rangle\left\langle\psi_{T}\right|\right]=\sum_{k=1}^{K} x_{k} T_{k}(\rho)=T(\rho),
$$

where, $E_{k}=|k\rangle\langle k|$ for all $k \in\{1, \ldots, K\}$. Thus, we showed that if $\mathcal{J}_{\mathcal{C}}$ is isomorphic to a polytope, there is a measure-and-prepare processor $\mathcal{P}$ that implements all $T \in \mathcal{C}$ exactly with program dimension $\operatorname{dim}\left(\mathcal{H}_{P}\right)=d_{P}=K$.

Conversely, let $\mathcal{P}$ be a measure-and-prepare $\mathrm{PQP}_{\mathcal{C}}$ with a $K$-outcome POVM. Then, $\mathcal{C}$ is the convex hull of at most $K$ extreme points, since by definition for any $T \in \mathcal{C}$

$$
T=\mathcal{P}\left(\cdot \otimes \pi_{T}\right)=\sum_{k=1}^{K} p_{k} T_{k}
$$

with $p_{k}=\operatorname{tr}\left[E_{k} \pi_{T}\right]$, i.e. $\left(p_{1}, \ldots, p_{K}\right)$ is a probability distribution. Thus, the extreme points of $\mathcal{C}$ are a subset of $\left\{T_{1}, \ldots, T_{K}\right\}$ and hence $\mathcal{C}$ is a polytope.

The following corollary assures that if we want to check whether channels $T \in \mathcal{T}_{U V}$ are programmable exactly by a measure-and-prepare $\mathrm{CPQP}_{U V}$ with finite-dimensional program register, we can consider the specific structure of the commutant $\mathcal{K}$.

Corollary 19. Let $U$ be an irrep of a compact group $G$ on $\mathcal{H}_{1}$ and $V$ a representation of $G$ on $\mathcal{H}_{2}$. Furthermore, let $\mathcal{K}$ be the commutant of $\bar{U} \otimes V$. Then, $\mathcal{K}$ is abelian if and only if there is a measure-and-prepare $C P Q P_{U V} \mathcal{P}$ that implements all $T \in \mathcal{T}_{U V}$ exactly with $d_{P} \leq K$, where $K$ is the number of irreps appearing in the direct sum decomposition of $\bar{U} \otimes V$.

Proof. The proof directly follows from Lemma 16 and Proposition 18.

We give an example of a group for which exact programmability holds with a measureand-prepare $\mathrm{CPQP}_{U V}$.

Example 20 (Unitarily covariant channels). We consider a measure-and-prepare $C P Q P_{U V}$ that implements all $U U$-covariant channels where $U$ is the defining representation of the group $G=\mathcal{U}_{1}$. The tensor representation $\bar{U} \otimes U$ can be decomposed into a direct sum of a one-dimensional and $a\left(d^{2}-1\right)$-dimensional irrep both with multiplicity 1 . Thus, elements of the commutant consist of a one-dimensional block with multiplicity equal to one and a one-dimensional block with multiplicity $d^{2}-1$, i.e. in this case $K=2, n_{1}=b_{1}=1, n_{2}=1$ and $b_{2}=d^{2}-1$. Due to Lemma 11, we know that the Choi-Jamiolkowski state $c_{T} \in \mathcal{J}_{U U}$ satisfies $\left[c_{T}, \bar{U}_{g} \otimes U_{g}\right]=0$. The state space therefore has the following form:

$$
\mathcal{K} \cap \mathcal{D}\left(\mathcal{H}_{1} \otimes \mathcal{H}_{1}\right)=\left\{\hat{\alpha} \frac{\mathbb{1}}{d^{2}}+(1-\hat{\alpha})|\Omega\rangle\left\langle\Omega|| \hat{\alpha} \in\left[0, \frac{d^{2}}{d^{2}-1}\right]\right\}\right.
$$


with extreme points $|\Omega\rangle\langle\Omega|$ and $\frac{1}{d^{2}-1}(\mathbb{1}-|\Omega\rangle\langle\Omega|)$. Thus, $T$ has the form $T(\cdot)=\hat{\alpha} \operatorname{tr}(\cdot) \frac{\mathbb{1}}{d}+$ $(1-\hat{\alpha})$ id [26, 40]. Every $c_{T}$ can be written as convex combination of the two extreme points and the set of all convex combinations

$$
\mathcal{K} \cap \mathcal{D}\left(\mathcal{H}_{1} \otimes \mathcal{H}_{1}\right)=\left\{x|\Omega\rangle\left\langle\Omega\left|+(1-x) \frac{1}{d^{2}-1}(\mathbb{1}-|\Omega\rangle\langle\Omega|)\right| x \in[0,1]\right\}\right.
$$

is isomorphic to a 1-simplex. Thus, the measure-and-prepare CPQP $P_{U V}$ can be implemented with program dimension $d_{P}=2$ using the construction in Proposition 18.

\subsection{Structure of the commutant of the tensor representation}

Based on the previous section, one could argue that in the case where the direct sum decomposition of $\bar{U} \otimes V$ consists of at least one one-dimensional irrep with multiplicity $n_{k}>1$, it would not be possible to implement the corresponding $U V$-covariant channel exactly in this case. It is instructive to see why this situation never arises, which we will discuss in this section.

The argument is the following: Assume $d_{1}=d_{2}=d$ and that there is a $k \in\{1, \ldots, K\}$ such that $n_{k}>1$. Then, there would be elements in $\mathcal{J}_{U V}$ of the form

$$
B_{\varphi}=\left|\varphi_{k}\right\rangle\left\langle\varphi_{k}\right| \oplus \mathbf{0} \in \mathcal{J}_{U V}
$$

where $\left|\varphi_{k}\right\rangle\left\langle\varphi_{k}\right| \in \mathcal{D}\left(\mathcal{H}_{k}^{\prime}\right)$ is a rank-one projector and $\operatorname{dim}(\mathbf{0})=d^{2}-n_{k}$. By the ChoiJamiołkowski isomorphism, there is a corresponding channel $T \in \mathcal{T}_{U V}$. We consider a Kraus representation of the channel $T$. Since $T$ is a completely positive map and $\operatorname{rank}\left(B_{\varphi}\right)=1$, the channel $T$ can be written as $T(\cdot)=X(\cdot) X^{*}$ with one Kraus operator $X \in \mathcal{B}\left(\mathcal{H}_{2}\right)$. Since, additionally, $T$ is trace-preserving, we know that $X X^{*}=\mathbb{1}=X^{*} X$, i.e. the Kraus operator is a unitary and $T$ is a unitary channel

$$
T(\cdot)=W(\cdot) W^{*}
$$

with $W \in \mathcal{U}_{2}$.

Since there are infinitely many pure states, the processor would have to implement infinitely many corresponding unitary channels. This contradicts the No-Programming Theorem according to which there is no processor that implements infinitely many unitary channels exactly with program dimension $d_{P}<\infty$. This shows that a processor $\mathcal{P}$ with $d_{P}<\infty$ cannot exist if $b_{k}=1$ and $n_{k}>1$ for some $k \in\{1, \ldots, K\}$. Note that the above argument fails for $b_{k}>1$, since then there might be no rank-1 elements in $\mathcal{K}$.

However, from a representation-theoretic perspective this situation does not arise because a one-dimensional irrep in the direct sum decomposition of $U \otimes \bar{V}$ always has multiplicity $\leq 1$ as the following proposition shows.

Proposition 21. Let $U$ be an irrep on $\mathcal{H}_{1}$ of a compact group $G$. Let $V$ be another representation of $G$ on $\mathcal{H}_{2}$ with dimension $d_{2} \leq d_{1}$. In a direct sum decomposition of $\bar{U} \otimes V$, the one-dimensional irreps $\lambda$ appear with multiplicity $n_{\lambda} \leq 1$.

Proof. Let $\chi_{U}$ be the character of the irrep $U, \chi_{V}$ the character of $V$, and $\lambda$ the character of the one-dimensional irrep $\lambda$. We use the following scalar product (see Lemma 8)

$$
\langle\chi, \psi\rangle=\int_{G} \overline{\chi(g)} \psi(g) d \mu(g),
$$

where $\mu$ is the Haar measure on $G$. Here, $\psi$ is the character of an arbitrary representation of $G$. Note that if $U$ is an irrep, then this scalar product of the corresponding characters 
gives the multiplicity of $U$ in the representation corresponding to $\psi$ (see Corollary 9). We want to show that the multiplicity of $\lambda$ in $\bar{U} \otimes V$ is $\leq 1$. Note that the character of $\bar{U} \otimes V$ is $\bar{\chi}_{U} \cdot \chi_{V}$. Let $\hat{G}$ be the set of irreps of $G$ and let

$$
\chi_{V}=\sum_{\alpha \in \hat{G}} n_{\alpha} \chi_{\alpha}
$$

be the decomposition of $V$ into irreducible representations on the level of characters. Then,

$$
\begin{aligned}
\left\langle\lambda, \bar{\chi}_{U} \cdot \chi_{V}\right\rangle & =\int_{G} \overline{\lambda(g)}\left(\overline{\chi_{U}(g)} \chi_{V}(g)\right) d \mu(g) \\
& =\int_{G} \overline{\lambda(g) \chi_{U}(g)} \chi_{V}(g) d \mu(g) \\
& =\left\langle\lambda \cdot \chi_{U}, \chi_{V}\right\rangle . \\
& =\sum_{\alpha \in \hat{G}} n_{\alpha}\left\langle\lambda \cdot \chi_{U}, \chi_{\alpha}\right\rangle .
\end{aligned}
$$

Since $\lambda$ is the character of a one-dimensional irrep, it is equal to the representation itself and $|\lambda(g)|^{2}=1$ for all $g \in G$. Thus, $\left\langle\lambda \cdot \chi_{U}, \lambda \cdot \chi_{U}\right\rangle=1$ and the representation corresponding to $\lambda \cdot \chi_{U}$ is again irreducible. Note that the representation corresponding to $\lambda \cdot \chi_{U}$ has the same dimension as $U$.

The scalar product $\left\langle\lambda \cdot \chi_{U}, \chi_{\alpha}\right\rangle$ thus gives the multiplicity of $\lambda \cdot \chi_{U}$ in $\chi_{\alpha}$. Let $\alpha$ be such that $n_{\alpha}>0$. If the dimension of the representation corresponding to $\chi_{\alpha}$ is smaller than $d_{2}$, then $\left\langle\lambda \cdot \chi_{U}, \chi_{\alpha}\right\rangle=0$ by Lemma 8. Thus, $\left\langle\lambda, \bar{\chi}_{U} \cdot \chi_{V}\right\rangle=0$. If the dimension is $d_{2}$, then $\chi_{V}=\chi_{\alpha}$ for dimensional reasons and $V$ is irreducible. The multiplicity of an irrep in another irrep can be at most equal to 1 which proves the assertion.

Remark 22. We can also give a direct algebraic argument for the impossibility of a onedimensional irrep of $G$ to occur in $\bar{U} \otimes V$ with multiplicity larger than one, if $U$ is an irrep and $V$ is an irrep, too, and has the same dimension as $U$. Namely, observe that all pure states in the multiplicity space $\mathcal{K}$ of a one-dimensional irrep have to be maximally entangled due to Schur's Lemma (their reduced states on both factors have to be maximally mixed). However, there is no two- or higher-dimensional subspace in $d \times d$ that merely consists of maximally entangled states (see, for instance Ref. [12, Proposition 6]).

After this discussion, we give a different construction of covariant programmable quantum processors which is more widely applicable.

\subsection{Covariant programmable quantum processors from teleportation}

If $\mathcal{K}$ is not abelian, we need a different method to show exact programmability. This case appears for example for the finite group $A_{4}[16$, p. 20], which is outside the scope of Ref. [30].

Example 23. The alternating group $A_{4}$ is a subgroup of the symmetric group $S_{4}$ consisting of only the even permutations:

$$
A_{4}=\{e,(12)(34),(13)(24),(14)(23),(123),(134),(243),(142),(321),(431),(342),(241)\} .
$$

Note that $K_{4}=\{e,(12)(34),(13)(24),(14)(23)\}$ is an abelian subgroup of $A_{4}$, in fact isomorphic to the Klein group, and since it can be described as the set of all elements of order at most 2 , it is a normal subgroup. Its cosets are $(123) K_{4}$ and $(321) K_{4}=(123)^{2} K_{4}$, which are conjugacy classes, and the quotient $A_{4} / K_{4}$ is the cyclic group $\mathbb{Z} / 3$ of three elements. 
The group has four irreps $\varphi^{(0)}, \varphi^{(1)}, \varphi^{(2)}$, and $\vartheta$. The first three, $\varphi^{(0)}, \varphi^{(1)}, \varphi^{(2)}$, are onedimensional, while $\vartheta$ is three-dimensional [16, Section 2.3]. Indeed, with the third root of unity $\zeta=e^{2 \pi i / 3}$, we have $\varphi^{(j)}\left((123)^{k} K_{4}\right)=\zeta^{j k}$. On the other hand, $\vartheta$ has a very intuitive form as the real $S O(3)$ symmetries of a regular tetrahedron in three-space: concretely, let $\mathcal{H}=(1,1,1,1)^{\perp}=\left\{\left(a_{1}, a_{2}, a_{3}, a_{4}\right): \sum_{i} a_{i}=0\right\} \subset \mathbb{C}^{4}$, which is spanned by the vertices $v_{1}=(3,-1,-1,-1), v_{2}=(-1,3,-1,-1), v_{3}=(-1,-1,3,-1), v_{4}=(-1,-1,-1,3)$ of the tetrahedron. The group action of $\vartheta$ is simply by permutation of the coordinate axes, and so all its matrices are real; as a consequence, $\bar{\vartheta}=\vartheta$.

Consider now the set of $\vartheta \vartheta$-covariant channels. Note that they form a semigroup (since they are closed under composition), and at the same time a closed convex set, isomorphic to the $3 \times 3$-states invariant under $\vartheta \otimes \vartheta$. The latter representation decomposes as

$$
\vartheta \otimes \vartheta \simeq \varphi^{(0)} \oplus \varphi^{(1)} \oplus \varphi^{(2)} \oplus 2 \vartheta,
$$

where $\vartheta$ appears with multiplicity 2. Hence, there are three one-dimensional blocks on the diagonal and two three-dimensional ones, i.e. the commutant consists of three onedimensional blocks and a two-dimensional block of multiplicity three. Thus, Lemma 16 shows that $J_{\vartheta \vartheta}$ is not a polytope; indeed its extreme points are three maximally entangled pure states, as well as a two-dimensional family of rank-three states, parametrized by the surface of the Bloch sphere. This implies that Corollary 19 does not cover all cases of interest.

We can give a more explicit description of the extreme points and the associated quantum channels. To start, because of the semigroup property, the unitary covariant channels form a group, and since by the preceding representation-theoretic analysis it has three elements, corresponding to the three maximally entangled pure states in $J_{\vartheta \vartheta}$, we are looking for a unitary $V$ of degree 3 that commutes with $\vartheta$ up to a phase given by the one-dimensional irreps: $\vartheta_{g} V \vartheta_{g}^{*}=\varphi_{g}^{(1)} V$. Then, the unitary channels $T_{j}(\rho)=V^{j} \rho V^{* j}(j=0,1,2)$ are $\vartheta \vartheta$-covariant. For instance, $V$ can be given as

$$
\begin{aligned}
& V\left|v_{1}\right\rangle=\frac{1}{2}\left(\quad\left|v_{2}\right\rangle+\zeta\left|v_{3}\right\rangle+\zeta^{2}\left|v_{4}\right\rangle\right), \\
& V\left|v_{2}\right\rangle=\frac{1}{2}\left(\left|v_{1}\right\rangle \quad+\zeta^{2}\left|v_{3}\right\rangle+\zeta\left|v_{4}\right\rangle\right), \\
& V\left|v_{3}\right\rangle=\frac{1}{2}\left(\zeta\left|v_{1}\right\rangle+\zeta^{2}\left|v_{2}\right\rangle+\left|v_{4}\right\rangle\right), \\
& V\left|v_{4}\right\rangle=\frac{1}{2}\left(\zeta^{2}\left|v_{1}\right\rangle+\zeta\left|v_{2}\right\rangle+\left|v_{3}\right\rangle \quad\right),
\end{aligned}
$$

keeping in mind that this description is redundant (but consistent), as $\left|v_{1}\right\rangle+\left|v_{2}\right\rangle+\left|v_{3}\right\rangle+$ $\left|v_{4}\right\rangle=0$ and the sum of the r.h.s. vectors vanishes, too. This defines a unitary because the pairwise inner product of any two of the r.h.s. vectors equals the inner product of the corresponding $\left|v_{i}\right\rangle$ on the l.h.s..

The remaining non-unitary, yet unital, channels, we have to find, are those whose ChoiJamiotkowski states are extreme points of rank 3 of $J_{\vartheta \vartheta}$, which means that the channels have Kraus rank 3. There is one distinguished element in this set, namely the WernerHolevo channel [43]

$$
W(\rho)=\frac{1}{2}\left((\operatorname{tr} \rho) \mathbb{1}-\rho^{T}\right),
$$

where $T$ denotes the transpose, whose Choi-Jamiotkowski state $\alpha$ is the normalized projector onto the $3 \times 3$-antisymmetric subspace. It is $\bar{U} U$-covariant for arbitrary $U \in S U(3)$; in particular it is $\vartheta \vartheta$-covariant if we recall that $\vartheta$ is real, but it is much more symmetric than 
that. It has the curious property that it is extremal in the set of all CPTP maps, hence in particular in the set of covariant ones. Moreover, we note that the channels $T_{j} \circ W \circ T_{k}$ $(j, k=0,1,2)$ are all $\vartheta \vartheta$-covariant by the semigroup property, giving further extreme points. Their Choi-Jamiotkowski states, which are $\left(\bar{V}^{k} \otimes V^{j}\right) \alpha\left(\bar{V}^{k} \otimes V^{j}\right)^{*}$, generate the qubit algebra $\mathcal{A}$ of the multiplicity space of $\vartheta$ in Eq. (3.1). In particular, we will find subsequently elements from the algebra which are a representation of the Pauli matrices $\sigma_{X}, \sigma_{Y}, \sigma_{Z}$. This was inspired by the approach taken in [14] for a different problem. Let $P:=\mathbb{1}_{9}-|\Omega\rangle\left\langle\Omega\left|-\left(\mathbb{1}_{3} \otimes V\right)\right| \Omega\right\rangle\left\langle\Omega\left|\left(\mathbb{1}_{3} \otimes V^{*}\right)-\left(\mathbb{1}_{3} \otimes V\right)^{2}\right| \Omega\right\rangle\langle\Omega|\left(\mathbb{1}_{3} \otimes V^{*}\right)^{2}$ be the projection onto $\mathcal{A}$. Using the form of $\alpha$, which is proportional to the projector onto the antisymmetric subspace, we infer that $P F \in \mathcal{A}$, where $F$ is the flip (or swap) operator on $\mathbb{C}^{3} \otimes \mathbb{C}^{3}$. Furthermore, we can verify that $\left[P, \mathbb{1}_{3} \otimes V\right]=0$ and $[P, F]=0$. Combining this with the previous finding that the Choi-Jamiotkowski states of $T_{j} \circ W \circ T_{k}$ are in $\mathcal{A}$, we obtain four linearly independent elements of $\mathcal{A}$, namely $P, P F, P\left(V \otimes V^{*}\right)$ and $P F\left(V \otimes V^{*}\right)$. Since $P F$ is traceless, $P$ is orthogonal to all elements except $P\left(V \otimes V^{*}\right)$ and $P F$ is orthogonal to all elements except $P F\left(V \otimes V^{*}\right)$. Thus, we can identify $P \simeq \mathbb{1}, V_{X}:=P F \simeq \sigma_{X}$ and make the ansatz $\sigma_{Y} \simeq V_{Y}:=a P F\left(V \otimes V^{*}\right)+b P F, \sigma_{Z}=-i \sigma_{X} \sigma_{Y} \simeq V_{Z}:=-i\left(a P\left(V \otimes V^{*}\right)+b P\right)$. Imposing $\operatorname{tr}\left(V_{X} V_{Y}\right)=0$ and $\operatorname{tr}\left(V_{Y}^{2}\right)=6$ yields $a=\frac{2}{\sqrt{3}}$ and $b=\frac{1}{\sqrt{3}}$ :

$$
\begin{aligned}
\mathbb{1} & \simeq V_{0}=P, \\
\sigma_{X} & \simeq V_{X}=P F, \\
\sigma_{Y} & \simeq V_{Y}=\frac{1}{\sqrt{3}} P F\left(2 V \otimes V^{*}+\mathbb{1}\right), \\
\sigma_{Z} & \simeq V_{Z}=\frac{1}{i \sqrt{3}} P\left(2 V \otimes V^{*}+\mathbb{1}\right) .
\end{aligned}
$$

We can verify that indeed $V_{i} V_{j}+V_{j} V_{i}=2 \delta_{i j} P$ for $i, j \in\{X, Y, Z\}$. Hence, we can use a Bloch ball representation for the Choi-Jamiotkowski states $J$ in $\mathcal{A}: J \in \mathcal{A}$ if and only if

$$
J=J_{\vec{\lambda}}=\frac{1}{6}\left(P+\lambda_{x} V_{X}+\lambda_{y} V_{Y}+\lambda_{z} V_{Z}\right),
$$

where $\vec{\lambda}=\left(\lambda_{x}, \lambda_{y}, \lambda_{z}\right) \in \mathbb{R}^{3}$ with $\lambda_{x}^{2}+\lambda_{y}^{2}+\lambda_{z}^{2} \leq 1$. Via the Choi-Jamiotkowski isomorphism, these describe $\vartheta \vartheta$-covariant channels $W_{\vec{\lambda}}$. The extremal ones are precisely the previously found $T_{0}=\mathrm{id}, T_{1}$ and $T_{2}$, as well as the $W_{\vec{\lambda}}$ with $\lambda_{x}^{2}+\lambda_{y}^{2}+\lambda_{z}^{2}=1$.

We can construct an exact quantum processor for covariant channels based on teleportation simulation, i.e. the simulation of quantum channels by quantum teleportation. In the case of the Pauli group this goes back to the Refs. [6, 7]. Important developments concerning the teleportation of covariant channels can be found in Refs. [9, 23, 31]. See also the very recent Ref. [41]. We know from Refs. [35, p. 58] and [45, Proposition 2] that it is always possible to simulate $U V$-covariant channels exactly using the corresponding Choi-Jamiołkowski state. This can easily be formulated as a processor which uses the Choi-Jamiołkowski state as program state and performs the teleportation protocol. Therefore, the dimension of the program register is $d_{1} d_{2}$. We will make this precise in the next proposition already present in Refs. [35, 45], which we have included here for convenience.

Proposition 24. Let $G$ be a compact group and let $U$ be an irreducible representation on $\mathcal{H}_{1}$. Let $V$ be another representation of $G$ on $\mathcal{H}_{2}$. Then, there is a $C P Q P_{U V}$ with program dimension $d_{P} \leq d_{1} d_{2}$.

Proof. Let $\rho \in \mathcal{D}\left(\mathcal{H}_{1}\right)$ be the state to be teleported. In this proof, we identify the input space as system $A, \mathcal{H}_{1} \simeq \mathcal{H}_{A}$, and the program as a composite system with parts $A^{\prime}$ and 
$B, \mathcal{H}_{P} \simeq \mathcal{H}_{A^{\prime}} \otimes \mathcal{H}_{B}=: \mathcal{H}_{A^{\prime} B}$, where $\mathcal{H}_{A^{\prime}} \simeq \mathcal{H}_{A}$ and $\mathcal{H}_{B} \simeq \mathcal{H}_{2}$ is isomorphic to the output space of $T$. We will write $d_{1}$ for the dimension of $A$. Note that $\operatorname{dim} A=\operatorname{dim} A^{\prime}=\operatorname{dim} A^{\prime \prime}=$ $d_{1}$. The Choi-Jamiołkowski states corresponding to the channels that are simulated serve as program states of the processor running the following protocol:

i) the processor measures according to the POVM

$$
\left\{M_{g}\right\}_{g \in G}:=\left\{d_{1}^{2}\left(\mathbb{1} \otimes \bar{U}_{g}\right)|\Omega\rangle\left\langle\left.\Omega\right|_{A A^{\prime}}\left(\mathbb{1} \otimes \bar{U}_{g}\right)^{*} d \mu(g)\right\}_{g \in G} .\right.
$$

Due to Schur's Lemma this is a POVM. Note that this POVM can potentially be continuous.

ii) On outcome $g$, apply $V_{g}^{*}(\cdot) V_{g}$ to the outcome of the protocol.

This construction of a processor implements $U V$-covariant channels $T \in \mathcal{T}_{U V}$ with the Choi-Jamiołkowski state as program state. The map is defined as

$$
\mathcal{P}(X \otimes Y):=\int_{G} V_{g}^{*} \operatorname{tr}_{A A^{\prime}}\left[(X \otimes Y)\left(M_{g} \otimes \mathbb{1}_{B}\right)\right] V_{g} \quad \forall X \in \mathcal{B}\left(\mathcal{H}_{A}\right), \forall Y \in \mathcal{B}\left(\mathcal{H}_{A^{\prime} B}\right)
$$

and extended by linearity. Let us verify that indeed

$$
\mathcal{P}\left(\rho^{A} \otimes c_{T}^{A^{\prime} B}\right)=T(\rho) .
$$

In the following, we insert the definition of the Choi-Jamiołkowski state $c_{T}^{A^{\prime} B}=\left(\operatorname{id}_{A^{\prime}} \otimes\right.$ $\left.T^{A^{\prime \prime} B}\right)|\Omega\rangle\left\langle\left.\Omega\right|_{A^{\prime} A^{\prime \prime}}\right.$ and we use $\left.(X \otimes \mathbb{1}) \mid \Omega\right\rangle=\left(\mathbb{1} \otimes X^{T}\right)|\Omega\rangle, X^{T}=\bar{X}$ for Hermitian $X$ as well as $\operatorname{tr}_{A^{\prime}}\left[(X \otimes \mathbb{1})|\Omega\rangle\left\langle\left.\Omega\right|_{A A^{\prime}}(Y \otimes \mathbb{1})\right]=1 / d_{1} X Y\right.$. Here, $A^{\prime \prime}$ is again a system isomorphic to $A$. We calculate

$$
\begin{aligned}
& \operatorname{tr}_{A A^{\prime}}\left[\left(\rho^{A} \otimes c_{T}^{A^{\prime} B}\right) M_{g} \otimes \mathbb{1}_{B}\right] \\
& =d_{1}^{2} d \mu(g) \operatorname{tr}_{A A^{\prime}}\left[\left(\rho^{A} \otimes\left(\operatorname{id}_{A^{\prime}} \otimes T^{A^{\prime \prime} B}\right)|\Omega\rangle\left\langle\left.\Omega\right|_{A^{\prime} A^{\prime \prime}}\right)\left(\mathbb{1}_{A} \otimes \bar{U}_{g}\right)|\Omega\rangle\left\langle\left.\Omega\right|_{A A^{\prime}}\left(\mathbb{1}_{A} \otimes \bar{U}_{g}\right)^{*} \otimes \mathbb{1}_{B}\right]\right.\right. \\
& =d_{1}^{2} d \mu(g) \operatorname{tr}_{A A^{\prime}}\left[\left(\left(\operatorname{id}_{A^{\prime}} \otimes T^{A^{\prime \prime} B}\right)|\Omega\rangle\left\langle\left.\Omega\right|_{A^{\prime} A^{\prime \prime}}\right)\left(\mathbb{1}_{A} \otimes \bar{U}_{g}\right)\left(\mathbb{1}_{A} \otimes \bar{\rho}^{A^{\prime}}\right)|\Omega\rangle\left\langle\left.\Omega\right|_{A A^{\prime}}\left(\mathbb{1}_{A} \otimes \bar{U}_{g}\right)^{*} \otimes \mathbb{1}_{B}\right]\right.\right. \\
& =d_{1}^{2} d \mu(g) \operatorname{tr}_{A^{\prime}}\left(\left(\operatorname{id}_{A^{\prime}} \otimes T^{A^{\prime \prime} B}\right)|\Omega\rangle\left\langle\left.\Omega\right|_{A^{\prime} A^{\prime \prime}}\left(\bar{U}_{g} \bar{\rho} \bar{U}_{g}^{*} \otimes \mathbb{1}_{B}\right)_{A^{\prime}}\right) \frac{1}{d_{1}}\right. \\
& =\frac{d_{1}}{d_{1}} T\left(U_{g} \rho U_{g}^{*}\right) d \mu(g) \\
& =V_{g} T(\rho) V_{g}^{*} d \mu(g),
\end{aligned}
$$

Applying the unitary conjugation as in Eq. (3.2) and integrating yields Eq. (3.3). This shows that $\mathcal{P}$ is indeed a $\mathrm{CPQP}_{U V}$ with the desired program dimension.

Using the structure of the commutant $\mathcal{K}$, we can reduce the program requirements further:

Theorem 25. Let $U$ be an irrep on $\mathcal{H}_{1}$ of a compact group $G, V$ another representation of $G$ on $\mathcal{H}_{2}$, and let $\mathcal{K}$ be of the form

$$
\mathcal{K}=\bigoplus_{k=1}^{K} \mathbb{1}_{b_{k}} \otimes \mathcal{B}\left(\mathcal{H}_{k}^{\prime}\right) .
$$

Then, there exists a $C P Q P_{U V}$ with program dimension $d_{P}=\sum_{k=1}^{K} n_{k}$, where $n_{k}=$ $\operatorname{dim}\left(\mathcal{H}_{k}^{\prime}\right)$. 
Proof. Since we have a programmable quantum processor $\mathcal{P}$, constructed in Proposition 24 , available, we combine its protocol, i.e. the teleportation simulation part, with a compression map which reduces the program dimension. Instead of $\mathcal{K}$ we consider a simpler matrix algebra with all multiplicities removed. These matrix algebras are isomorphic. Let

$$
D^{*}: \mathcal{K} \rightarrow \bigoplus_{k=1}^{K} \mathcal{B}\left(\mathcal{H}_{k}^{\prime}\right)
$$

be this isomorphism and let $\mathcal{H}_{P}=\bigoplus_{k=1}^{K} \mathcal{H}_{k}^{\prime}$, which has dimension $d_{P}=\sum_{k=1}^{K} n_{k}$. Thus, $D^{*}$ is a unital completely positive map with unital completely positive inverse $C^{*}[8$, Example II.6.9.3(i)]. Let $\tilde{C}, \tilde{D}$ be their dual maps. Both maps can be extended to quantum channels $C: \mathcal{B}\left(\mathcal{H}_{1} \otimes \mathcal{H}_{2}\right) \rightarrow \mathcal{B}\left(\mathcal{H}_{P}\right), D: \mathcal{B}\left(\mathcal{H}_{P}\right) \rightarrow \mathcal{B}\left(\mathcal{H}_{1} \otimes \mathcal{H}_{2}\right)$ by composing them with trace-preserving conditional expectations onto the respective subalgebras. Note that we identify the subalgebras in the images of $\tilde{C}, \tilde{D}$ with subalgebras of $\mathcal{B}\left(\mathcal{H}_{P}\right)$ and $\mathcal{B}\left(\mathcal{H}_{1} \otimes \mathcal{H}_{2}\right)$, respectively. The maps $C$ and $D$ are completely positive since the conditional expectations are [5, Proposition 5.2.2]. We define

$$
\mathcal{P}^{\prime}=\mathcal{P} \circ(\text { id } \otimes D),
$$

and it follows that $\mathcal{P}^{\prime}\left(\rho \otimes C\left(c_{T}\right)\right)=T(\rho)$.

Remark 26. Comparing Proposition 24 to Theorem 25, we see that the bound in the theorem is strictly better as soon as there is one index $k$ such that $b_{k}>1$. Recall that the $b_{k}$ are the dimensions of the irreps appearing in $\bar{U} \otimes V$. Thus, the improvement of Theorem 25 over Proposition 24 is larger, the more different irreps of dimension strictly larger than 1 appear in $\bar{U} \otimes V$ and the larger the dimension of these irreps is.

\section{Bounds for Approximate Programmability}

Since exact universal programmable quantum processors are impossible, there is a great interest in approximate versions of such devices. After having considered exact programmability in the last section, we now want to approximate the output such that the programmed output is close to the ideal output. Therefore, we need the concept of an $\epsilon$-CPQP $\mathrm{C}_{U V}$ from Definition 12, which is a processor that implements a channel $T^{\epsilon}$ instead of the exact result $T$. However, it should be $\epsilon$-close to the ideal one in diamond norm. Having recalled this, the question of program requirements arises and hence, we show that the program register

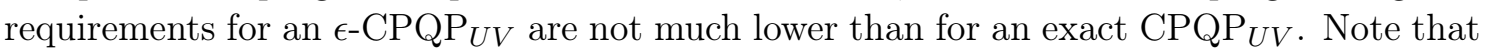
we can still benefit from the covariance property and the corresponding structure of the commutant $\mathcal{K}$.

\subsection{Upper bounds on the program dimension}

We construct generic upper bounds for the dimension of the program register $d_{P}$. We seek to establish an $\epsilon$-net on the set of covariant channels $\mathcal{T}_{U V}$. Therefore, we need the following result about $\epsilon$-nets from Ref. [28].

Lemma 27 ( $\epsilon$-nets in $\mathbb{R}^{n}[28$, Lemma 9.5]). Let $\epsilon \in(0,1)$ and let $\|\cdot\|$ be any norm on $\mathbb{R}^{n}$. There is an $\epsilon$-net $\mathcal{S}$ on the unit sphere $S_{\|\cdot\|}^{n-1}$ of $\left(\mathbb{R}^{n},\|\cdot\|\right)$ of cardinality

$$
|\mathcal{S}| \leq\left(1+\frac{2}{\epsilon}\right)^{n}
$$

That means, for all $x \in S_{\|\cdot\|}^{n-1}$, there is a $y \in \mathcal{S}$ such that $\|x-y\| \leq \epsilon$. 
Due to the Choi-Jamiołkowski isomorphism, there is a $c_{T} \in \mathcal{J}_{U V}$ corresponding to each $T \in \mathcal{T}_{U V}$. According to Lemma 11, we know that $\mathcal{J}_{U V} \subseteq \mathcal{K} \cap \mathcal{D}\left(\mathcal{H}_{1} \otimes \mathcal{H}_{2}\right)$. Thus, we can make use of the special block-diagonal structure of $\mathcal{K}$.

Proposition 28. Let

$$
\mathcal{K}=\bigoplus_{k=1}^{K} \mathbb{1}_{b_{k}} \otimes \mathcal{B}\left(\mathcal{H}_{k}^{\prime}\right) .
$$

For $\epsilon \in(0,1)$, there is a set $\mathcal{S}_{U V} \subseteq \mathcal{T}_{U V}$ such that for all $T \in \mathcal{T}_{U V}$, there is a $T^{\epsilon} \in \mathcal{S}_{U V}$ such that $\left\|T-T^{\epsilon}\right\|_{\diamond} \leq 2 \epsilon$. Moreover,

$$
\left|\mathcal{S}_{U V}\right| \leq\left(1+\frac{2}{\epsilon}\right)^{d_{n}}
$$

where $d_{n}=\sum_{k=1}^{K} n_{k}^{2}$.

Proof. Since $\mathcal{J}_{U V} \subseteq \mathcal{K}$ by Lemma 11 , the real vector space $\operatorname{Lin}_{\mathbb{R}} \mathcal{J}_{U V}$ generated by $\mathcal{J}_{U V}$ has dimension at most $d_{n}$. Using the Choi-Jamiołkowski isomorphism, we infer that $\operatorname{Lin}_{\mathbb{R}} \mathcal{T}_{U V}$ is a real subspace of $\mathcal{B}\left(\mathcal{B}\left(\mathcal{H}_{1}\right), \mathcal{B}\left(\mathcal{H}_{2}\right)\right)$ of dimension at most $d_{n}$. The restriction of the diamond norm turns $\left(\operatorname{Lin}_{\mathbb{R}} \mathcal{T}_{U V},\|\cdot\|_{\diamond}\right)$ into a real normed space which is isometrically isomorphic to $\left(\mathbb{R}^{d_{n}},\|\cdot\|\right)$ for some induced norm $\|\cdot\|$. Moreover, $\|T\|_{\diamond}=1$ for all $T \in \mathcal{T}_{U V}$. Lemma 27 thus ensures the existence of an $\epsilon$-net $\mathcal{S}$ on the unit sphere of $\left(\operatorname{Lin}_{\mathbb{R}} \mathcal{T}_{U V},\|\cdot\|_{\diamond}\right)$ and the unit sphere contains $\mathcal{T}_{U V}$. To obtain $\mathcal{S}_{U V}$, we repeat the following steps. Take $\Phi \in \mathcal{S}$. If $\Phi \in \mathcal{T}_{U V}$, keep it and proceed to the next element. If there is no $T \in \mathcal{T}_{U V}$ such that $\|\Phi-T\|_{\diamond} \leq \epsilon$, remove $\Phi$ from the set and proceed to the next element. If there is a $T \in \mathcal{T}_{U V}$ such that $\|\Phi-T\|_{\diamond} \leq \epsilon$, exchange $\Phi$ by $T$ and proceed to the next element. This algorithm constructs $\mathcal{S}_{U V}$ with the desired properties. Indeed, for any $T \in \mathcal{T}_{U V}$, there is a $\Phi \in \mathcal{S}$ and a $\mathcal{T}^{\epsilon} \in \mathcal{S}_{U V}$ such that $\|T-\Phi\|_{\diamond} \leq \epsilon,\left\|T^{\epsilon}-\Phi\right\|_{\diamond} \leq \epsilon$. Thus, $\left\|T^{\epsilon}-T\right\|_{\diamond} \leq 2 \epsilon$. The cardinality bound follows since by construction $\left|\mathcal{S}_{U V}\right| \leq|\mathcal{S}|$.

The previous proposition allows us to construct an $\epsilon$-CPQP $\mathrm{CV}_{V}$.

Proposition 29. For a compact group $G$ and representations $U$ on $\mathcal{H}_{1}, V$ on $\mathcal{H}_{2}$ such that

$$
\mathcal{K}=\bigoplus_{k=1}^{K} \mathbb{1}_{b_{k}} \otimes \mathcal{B}\left(\mathcal{H}_{k}^{\prime}\right),
$$

there exists an $\epsilon-C P Q P_{U V}$ with program dimension

$$
d_{P} \leq\left(1+\frac{2}{\epsilon}\right)^{d_{n}}
$$

where $d_{n}=\sum_{k=1}^{K} n_{k}^{2}$ and $n_{k}=\operatorname{dim}\left(\mathcal{H}_{k}^{\prime}\right)$.

Proof. Let $\mathcal{S}_{U V}=\left\{T_{1}^{\epsilon}, \ldots, T_{s}^{\epsilon}\right\}$ be the set from Proposition 28. Then, we can define a processor by

$$
\mathcal{P}(X \otimes Y)=\sum_{i=1}^{s}\langle i|Y| i\rangle T_{i}^{\epsilon}(X) \quad \forall X \in \mathcal{B}\left(\mathcal{H}_{1}\right), \forall Y \in \mathcal{B}\left(\mathcal{H}_{P}\right)
$$

and extending by linearity. Here, $\{|i\rangle\}_{i \in\{1, \ldots, s\}}$ is an orthonormal basis of $\mathcal{H}_{P}$. Choosing the program state for any $T \in \mathcal{T}_{U V}$ to be $|i\rangle\langle i|$ if $\left\|T-T_{i}^{\epsilon}\right\|_{\diamond} \leq 2 \epsilon$, the map $\mathcal{P}$ can be checked to be an $\epsilon-\mathrm{CPQP}_{U V}$ using Proposition 28. 
Remark 30. Note that in the case in which $U$ and $V$ are the trivial representation and $d_{1}=d_{2}=d$, Proposition 29 states that

$$
d_{P} \leq\left(1+\frac{2}{\epsilon}\right)^{d^{4}}
$$

This does not match the upper bounds of $d_{P} \leq(K / \epsilon)^{d^{2}}$ obtained in Ref. [27], which are also derived using $\epsilon$-nets. The reason is that the construction in Ref. [27] only implements all unitary channels in dimension d instead of all quantum channels, which is what our construction does. In the same setting, a slight arithmetic improvement of the work [47] yields lower bounds of the form

$$
d_{P} \geq\left(1+\frac{\Theta\left(d^{-2}\right)}{\sqrt{\epsilon}}\right)^{d^{2}-1-\delta}
$$

if the program states are required to be pure, for any $\delta>0$. Thus, the question remains if the bounds in Proposition 29 are optimal for trivial symmetries. We will argue that this is not the case.

By Ref. [10], the extreme points of $\operatorname{CPTP}\left(\mathcal{H}_{1}, \mathcal{H}_{2}\right)$ have Kraus rank of at most $d_{1}$, whereas general channels can have Kraus rank up to $d_{1} d_{2}$. The set of $\operatorname{CPTP}\left(\mathcal{H}_{1}, \mathcal{H}_{2}\right)$ is a subset of a real vector space of dimension $d_{1}^{2}\left(d_{2}^{2}-1\right)$, thus by Carathéodory's theorem, any channel is a combination of at most $r=d_{1}^{2}\left(d_{2}^{2}-1\right)+1$ extremal channels (see also Remark 13). Thus, we only need an $\epsilon$-net on the set of channels with Kraus rank at most $d_{1}$. Let $N$ be the cardinality of this net. Then, we can approximately program all extremal channels with a measure-and-prepare processor and pure orthogonal states in an $\mathrm{N}$-dimensional program register. This implies that the same processor approximately programs all channels by using mixtures of up to $r$ basis states as program registers; alternatively, we could use the corresponding superpositions of the basis vectors to obtain pure program states. It remains to bound $N$.

We can construct the $\epsilon$-net on the set of channels with Kraus rank at most $d_{1}$ by considering their Choi-Jamiotkowski states, which are states on $\mathcal{H}_{1} \otimes \mathcal{H}_{2}$ of rank $\leq d_{1}$. These can be purified to pure states on $\mathcal{H}_{1} \otimes \mathcal{H}_{2} \otimes \mathcal{H}_{1}^{\prime}$, where $\mathcal{H}_{1}^{\prime} \simeq \mathcal{H}_{1}$. In Ref. [18, Lemma III.6] it is shown that a $\delta$-net on such states with respect to the trace norm exists that has $N \leq$ $\left(\frac{5}{\delta}\right)^{2 d_{1}^{2} d_{2}}$ elements; tracing out $\mathcal{H}_{1}^{\prime}$ gives us the desired net on Choi-Jamiotkowski states with bounded rank, since the trace norm satisfies data-processing. By Ref. [42, Eq. (3.414)], for two channels $T_{1}, T_{2} \in \operatorname{CPTP}\left(\mathcal{H}_{1}, \mathcal{H}_{2}\right)$ and their respective Choi-Jamiolkowski states $c_{1}, c_{2}$, it holds that

$$
\left\|T_{1}-T_{2}\right\|_{\diamond} \leq d_{1}\left\|c_{1}-c_{2}\right\|_{1} .
$$

Thus, choosing $\delta=\frac{\epsilon}{d_{1}}$ provides the desired $\epsilon$-net.

We conclude that Proposition 29 is not optimal, because it gives an exponent of $d^{4}$, whereas the above construction improves this to $O\left(d^{3} \log d\right)$. It remains open, however, if the latter is optimal or whether it is possible to further improve this exponent. Note that for mixed program states, the bound in Eq. (4.1) does not apply, but we can purify the program states at only little extra cost, cf. Remark 13.

\subsection{Lower Bounds for the program dimension}

We seek to provide lower bounds on the program dimension of an $\epsilon$-CPQP $\mathrm{C}_{U V}$. The main idea is that all information about the $U V$-covariant channel $T \in \mathcal{T}_{U V}$ is contained in its 
corresponding Choi-Jamiołkowski state $c_{T}$. Thus, the program state $\pi_{T} \in \mathcal{D}\left(\mathcal{H}_{P}\right)$ has to store all information about $c_{T}$. Using the Holevo information to quantify the amount of information, we obtain the following lower bounds:

Theorem 31. Let $\epsilon \in[0,1), \mathcal{P}^{\epsilon} \in C P T P\left(\mathcal{H}_{1} \otimes \mathcal{H}_{P}, \mathcal{H}_{2}\right)$ be an $\epsilon-C P Q P_{U V}, G$ be a compact group with an irrep $U$ on $\mathcal{H}_{1}$ and $V$ be a representation on $\mathcal{H}_{2}$. Then, the following lower bound for the program dimension $d_{P}$ holds

$$
\frac{1}{2^{2 h(\epsilon)}}\left(\sum_{k=1}^{K} n_{k}\right)^{(1-2 \epsilon)}
$$

with $h(\epsilon)=H(\epsilon, 1-\epsilon)=-\epsilon \log \epsilon-(1-\epsilon) \log (1-\epsilon)$ being the binary entropy, $n_{k}$ the multiplicity of the irrep $k \in\{1, \ldots, K\}$ in the direct sum decomposition of $\bar{U} \otimes V$ and $\epsilon$ the approximation parameter of the $\epsilon-C P Q P_{U V}$.

Proof. Every channel $T^{\epsilon}$, the processor is able to implement, corresponds to a ChoiJamiołkowski state $c_{T^{\epsilon}} \in \mathcal{D}\left(\mathcal{H}_{1} \otimes \mathcal{H}_{2}\right)$ which can be understood as the output state of the processor $\mathcal{P}^{\epsilon} \in \operatorname{CPTP}\left(\mathcal{H}_{1} \otimes \mathcal{H}_{P}, \mathcal{H}_{2}\right)$ tensorized with the $d_{1}$-dimensional identity map id $: \mathcal{B}\left(\mathcal{H}_{1}\right) \rightarrow \mathcal{B}\left(\mathcal{H}_{1}\right)$. The construction

$$
\left[\mathrm{id} \otimes \mathcal{P}^{\epsilon}\right]\left(|\Omega\rangle\left\langle\left.\Omega\right|_{\mathcal{H}_{1} \otimes \mathcal{H}_{1}} \otimes \cdot\right): \mathcal{B}\left(\mathcal{H}_{P}\right) \rightarrow \mathcal{B}\left(\mathcal{H}_{1} \otimes \mathcal{H}_{2}\right)\right.
$$

provides a quantum channel which maps every program state $\pi_{T}$ to a Choi-Jamiołkowski state $c_{T^{\epsilon}}$. This is a completely positive map because the processor map itself and the identity map are completely positive.

Let

$$
\chi\left(\left\{\rho_{i}, p_{i}\right\}\right):=S\left(\sum_{i} p_{i} \rho_{i}\right)-\sum_{i} p_{i} S\left(\rho_{i}\right)
$$

be the Holevo information, where $S$ is the von Neumann entropy and $\left\{\rho_{i}, p_{i}\right\}$ is an ensemble of quantum states. Let $p^{(k)}:=\left(p_{1}^{(k)}, \ldots, p_{N_{k}}^{(k)}\right)$ be a probability distribution and let $\left|\psi_{j}^{(k)}\right\rangle\left\langle\psi_{j}^{(k)}\right| \in \mathcal{D}_{P}\left(\mathcal{H}_{k}^{\prime}\right), j \in\left\{1, \ldots, N_{k}\right\}$, be a collection of states such that $\left\{\left|\psi_{j}^{(k)}\right\rangle, p_{j}^{(k)}\right\}$ is a 1-design $[2,17]$, i.e. its average is the same as the uniform average over pure states with respect to the Haar measure. Then, $p=\left(\lambda_{1} p^{(1)}, \ldots, \lambda_{K} p^{(K)}\right)$ is a probability distribution on Choi-Jamiołkowski states $c_{T} \in \mathcal{J}_{U V}$, where $p_{j}^{(k)}$ corresponds to $c_{T_{(k), j}}:=0 \oplus \frac{1}{b_{k}} \mathbb{1}_{b_{k}} \otimes\left|\psi_{j}^{(k)}\right\rangle\left\langle\psi_{j}^{(k)}\right| \oplus 0$. Here, $\lambda_{k}=\frac{n_{k}}{d_{c}}$, where $d_{c}=\sum_{k=1}^{K} n_{k}$. The $c_{T_{(k), j}}$ are constructed such that they are elements of $\mathcal{J}_{\mathcal{U} V}$ and their structure follows from the form of $\mathcal{K}$. It can be checked that the average state is

$$
\frac{1}{d_{c}} \bigoplus_{k=1}^{K} \frac{\mathbb{1}_{b_{k}}}{b_{k}} \otimes \mathbb{1}_{n_{k}} .
$$

The probability distribution also induces ensembles $\left\{c_{T_{(k), j}^{\epsilon},}, p_{j}^{(k)}\right\}$ and $\left\{\pi_{T_{(k), j}}, p_{j}^{(k)}\right\}$.

Applying the data processing inequality [44, Section 10.7.2] (since the Holevo information is a mutual information), we get

$$
\chi\left(\left\{\pi_{T_{(k), j}}, p_{j}^{(k)}\right\}\right) \geq \chi\left(\left\{c_{T_{(k), j}^{\epsilon}}, p_{j}^{(k)}\right\}\right) .
$$

Furthermore, note that the processor implements $T$ up to accuracy $\epsilon$, i.e.

$$
\frac{1}{2}\left\|T-T^{\epsilon}\right\|_{\diamond} \leq \epsilon
$$


which can be related to the corresponding Choi-Jamiołkowski states [42, eq. 3.414]

$$
\frac{1}{2}\left\|c_{T}-c_{T}^{\epsilon}\right\|_{1} \leq \frac{1}{2}\left\|T-T^{\epsilon}\right\|_{\diamond} \leq \epsilon .
$$

Note that $c_{T}$ has a block-diagonal structure inherited from

$$
\mathcal{K}=\bigoplus_{k=1}^{K} \mathbb{1}_{b_{k}} \otimes \mathcal{B}\left(\mathcal{H}_{k}^{\prime}\right) .
$$

Since $\bigoplus_{k=1}^{K} \mathcal{B}\left(\mathcal{H}_{k}^{\prime}\right)$ and $\mathcal{K}$ are isomorphic as matrix algebras, extending the dual map of this isomorphism to a map $C: \mathcal{B}\left(\mathcal{H}_{1} \otimes \mathcal{H}_{2}\right) \rightarrow \mathcal{B}\left(\mathcal{H}_{d_{c}}\right)$ (as in the proof of Theorem 25),

$$
C: \bigoplus_{k=1}^{K} \mathbb{1}_{b_{k}} \otimes B_{k} \mapsto \bigoplus_{k=1}^{K} b_{k} B_{k}
$$

discards the multiplicity spaces and thus reduces the dimensions from $d_{1} d_{2}$ to $d_{c}=$ $\sum_{k=1}^{K} n_{k}$. Since the trace distance is contractive under quantum channels,

$$
\frac{1}{2}\left\|C\left(c_{T}\right)-C\left(c_{T^{\epsilon}}\right)\right\|_{1} \leq \frac{1}{2}\left\|c_{T}-c_{T^{\epsilon}}\right\|_{1} \leq \epsilon .
$$

With this trace-norm distance, we apply the Alicki-Fannes-Winter (AFW) inequality [3, 34], [46, Lemma 1] to bound the difference of the corresponding Holevo informations:

$$
\begin{aligned}
& \left|\chi\left(\left\{C\left(c_{T_{(k), j}}\right), p_{j}^{(k)}\right\}\right)-\chi\left(\left\{C\left(c_{T_{(k), j}^{\epsilon}}\right), p_{j}^{(k)}\right\}\right)\right| \\
& =\left|S\left(\sum_{k j} C\left(c_{T_{(k), j}}\right) p_{j}^{(k)}\right)-\sum_{k j} S\left(C\left(c_{T_{(k), j}}\right)\right) p_{j}^{(k)}-S\left(\sum_{k j} C\left(c_{\left.T_{(k), j}^{\epsilon}\right)}\right) p_{j}^{(k)}\right)+\sum_{k j} S\left(C\left(c_{T_{(k), j}^{\epsilon}}\right)\right) p_{j}^{(k)}\right| \\
& \leq\left|S\left(\sum_{k j} C\left(c_{T_{(k), j}}\right) p_{j}^{(k)}\right)-S\left(\sum_{k j} C\left(c_{T_{(k), j}^{\epsilon}}\right) p_{j}^{(k)}\right)\right|+\sum_{k j}\left|S\left(C\left(c_{T_{(k), j}}\right)\right)-S\left(C\left(c_{T_{(k), j}^{\epsilon}}^{\epsilon}\right)\right)\right| p_{j}^{(k)} \\
& \leq \epsilon \log d_{c}-h(\epsilon)+\epsilon \log d_{c}-h(\epsilon) \\
& =2 \epsilon \log d_{c}-2 h(\epsilon),
\end{aligned}
$$

with $h(\epsilon)=H(\epsilon, 1-\epsilon)=-\epsilon \log \epsilon-(1-\epsilon) \log (1-\epsilon)$ the binary entropy, where we used the AFW inequality in the last step.

Thus, we obtain

$$
\begin{aligned}
\chi\left(\left\{\pi_{T_{(k), j}}, p_{j}^{(k)}\right\}\right) & \geq \chi\left(\left\{c_{T_{(k), j}^{\epsilon}}, p_{j}^{(k)}\right\}\right) \\
& \geq \chi\left(\left\{C\left(c_{T_{(k), j}^{\epsilon}}\right), p_{j}^{(k)}\right\}\right) \\
& \geq \chi\left(\left\{C\left(c_{T_{(k), j}}\right), p_{j}^{(k)}\right\}\right)-2 \epsilon \log d_{c}-2 h(\epsilon) .
\end{aligned}
$$

Let us consider the term $\chi\left(\left\{C\left(c_{T_{(k), j}}\right), p_{j}^{(k)}\right\}\right)$. Since the $C\left(c_{T_{(k), j}}\right)$ of this ensemble are pure states, the second term of the Holevo information is zero. Moreover

$$
\sum_{k j} C\left(c_{T_{(k), j}}\right) p_{j}^{(k)}=\frac{\mathbb{1}_{d_{c}}}{d_{c}}
$$


and the von Neumann entropy of a maximally mixed state is $S\left(\frac{\mathbb{1}_{d_{c}}}{d_{c}}\right)=\log d_{c}$. Together with the inequality $\log d_{P} \geq \chi\left(\left\{\pi_{T_{(k), j}}, p_{j}^{(k)}\right\}\right)$, we obtain

$$
\begin{aligned}
\log d_{P} & \geq \chi\left(\left\{\pi_{T_{(k), j}}, p_{j}^{(k)}\right\}\right) \\
& \geq(1-2 \epsilon) \log d_{c}-2 h(\epsilon) \\
& =\log d_{c}^{(1-2 \epsilon)}-2 h(\epsilon) .
\end{aligned}
$$

This yields

$$
d_{P} \geq \frac{1}{2^{2 h(\epsilon)}} d_{c}^{(1-2 \epsilon)}=\frac{1}{2^{2 h(\epsilon)}}\left(\sum_{k=1}^{K} n_{k}\right)^{(1-2 \epsilon)},
$$

which proves the assertion.

The bound on $d_{P}$ we have derived increases with the block dimension $n_{k}$.

\section{Discussion}

In this section, we discuss our findings and mention some potential future work. We start by pointing out that Theorem 25 is in fact optimal. Note that for $\epsilon=0$ we obtain exact lower bounds from Theorem 31. Combining them with the teleportation protocol in Theorem 25, the optimal program requirements $d_{P}$ for an $\mathrm{CPQP}_{U V}$ are thus

$$
d_{c}=\sum_{k=1}^{K} n_{k}=d_{P}
$$

If we insisted on pure program states, we would get $d_{P}=d_{c}^{2}$ from Theorem 25 , replacing $\pi_{T}$ by a suitable purification (for example the canonical purification $\sqrt{d_{P}}\left(\mathbb{1} \otimes \pi_{T}^{1 / 2} W\right)|\Omega\rangle$, where $W$ is a suitable unitary). More refined constructions have been discussed in Remark 13. We leave it as an open question whether the upper bounds can be improved to match the lower ones also for pure program states. In the case where the commutant $\mathcal{K}$ is abelian, Corollary 19 answers the question in the affirmative.

In the present paper, we have shown that the problem of finding programmable quantum processors for $U V$-covariant channels for irreducible $U$ is very different from the situation concerning universal programmable quantum processors. While the former is always possible exactly with finite-dimensional program register, the latter is only possible approximately with finite-dimensional program register and requires a large program dimension. The question remains whether there are intermediate situations: Is there a group $G$ and a reducible representation $U$ such that there exists an exact $\mathrm{CPQP}_{U V}$ with finite-dimensional program register? If so, what determines if there are exact $\mathrm{CPQP}_{U V}$ with finite-dimensional program register? A major roadblock to extend our results to the case in which $U$ is no longer irreducible is Lemma 15, since we can no longer guarantee that all elements in $\mathcal{K} \cap \mathcal{D}\left(\mathcal{H}_{1} \otimes \mathcal{H}_{2}\right)$ correspond to trace-preserving maps.

A further question is, how upper bounds for $\epsilon>0$ can be constructed because the $\epsilon$ nets used in Ref. [27] and the general-purpose result Proposition 29, for instance, rely on orthogonal program states. Remark 30 already shows that the proposition is suboptimal for trivial symmetries. If $U$ is irreducible it can be outperformed applying the teleportation protocol as we saw in Section 3.3. Hence, we have good exact upper bounds and for an approximation in the case where the program states are not orthogonal, a different way to compress the program states is required. Therefore, the question arises whether we 
can find approximate upper bounds which improve the exact ones from Theorem 25 for $U$ irrep. However, Theorem 31 shows us that there is not much space for improvement using approximate processors.

\section{Acknowledgements}

AB and MG thank Matthias Christandl, Frank Himstedt, Alexander Müller-Hermes, Ion Nechita, Jitendra Prakash, Cambyse Rouzé, Daniel Stilck-França, Simone Warzel and Michael Wolf for discussions. AB acknowledges support from the VILLUM FONDEN via the QMATH Centre of Excellence (Grant no. 10059) and from the QuantERA ERA-NET Cofund in Quantum Technologies implemented within the European Union's Horizon 2020 Programme (QuantAlgo project) via the Innovation Fund Denmark. MG is funded by the Deutsche Forschungsgemeinschaft (DFG, German Research Foundation) under Germany's Excellence Strategy - EXC-2111 - 390814868. AW acknowledges financial support by the Spanish MINECO (projects FIS2016-86681-P and PID2019-107609GB-I00) with the support of FEDER funds, and the Generalitat de Catalunya (project CIRIT 2017-SGR-1127).

\section{References}

[1] M. Al Nuwairan. SU(2)-Irreducibly covariant and EPOSIC channels. arXiv:1306.5321, art. arXiv:1306.5321, 2013. URL https://arxiv.org/abs/1306.5321.

[2] A. Ambainis and J. Emerson. Quantum t-designs: t-wise independence in the quantum world. In Twenty-Second Annual IEEE Conference on Computational Complexity (CCC'07), pages 129-140, 2007. DOI: 10.1109/CCC.2007.26.

[3] K. M. R. Audenaert. A sharp continuity estimate for the von Neumann entropy. Journal of Physics A: Mathematical and Theoretical, 40(28):8127-8136, 2007. DOI: 10.1088/1751-8113/40/28/s18.

[4] L. Banchi, J. Pereira, S. Lloyd, and S. Pirandola. Convex optimization of programmable quantum computers. npj Quantum Information, 6(1):42, 2020. DOI: 10.1038/s41534-020-0268-2.

[5] F. Benatti. Dynamics, Information and Complexity in Quantum Systems. Springer, 2009. DOI: 10.1007/978-1-4020-9306-7.

[6] C. H. Bennett, G. Brassard, C. Crépeau, R. Jozsa, A. Peres, and W. K. Wootters. Teleporting an unknown quantum state via dual classical and Einstein-PodolskyRosen channels. Physical Review Letters, 70:1895-1899, 1993. DOI: 10.1103/PhysRevLett.70.1895.

[7] C. H. Bennett, D. P. Divincenzo, J. A. Smolin, and W. K. Wootters. Mixed-state entanglement and quantum error correction. Physical Review A, 54(5):3824-3851, 1996. DOI: 10.1103/PhysRevA.54.3824.

[8] B. Blackadar. Operator Algebras: Theory of $C^{*}$-Algebras and Von Neumann Algebras, volume 13 of Encyclopaedia of Mathematical Sciences. Springer, 2006. DOI: 10.1007/3540-28517-2.

[9] G. Chiribella, G. M. D'Ariano, and P. Perinotti. Realization schemes for quantum instruments in finite dimensions. Journal of Mathematical Physics, 50(4):042101042101, 2009. DOI: 10.1063/1.3105923.

[10] M.-D. Choi. Completely positive maps on complex matrices. Linear Algebra and Its Applications, 10:285-290, 1975. DOI: 10.1016/0024-3795(75)90075-0.

[11] C. Cîrstoiu, K. Korzekwa, and D. Jennings. Robustness of Noether's principle: Maximal disconnects between conservation laws and symmetries in quantum theory. Physical Review X, 10:041035, 2020. DOI: 10.1103/PhysRevX.10.041035. 
[12] T. Cubitt, A. Montanaro, and A. Winter. On the dimension of subspaces with bounded Schmidt rank. Journal of Mathematical Physics, 49(2):022107, 2008. DOI: $10.1063 / 1.2862998$.

[13] G. M. D'Ariano and P. Perinotti. Efficient universal programmable quantum measurements. Physical Review Letters, 94(9):090401, 2005. DOI: 10.1103/physrevlett.94.090401.

[14] T. Eggeling and R. F. Werner. Separability properties of tripartite states with $U \otimes U \otimes U$ symmetry. Physical Review A, 63:042111, 2001. DOI: 10.1103/PhysRevA.63.042111.

[15] M. Fannes, B. Haegeman, M. Mosonyi, and D. Vanpeteghem. Additivity of minimal entropy output for a class of covariant channels. arXiv:quant-ph/0410195, 2004. URL https://arxiv.org/abs/quant-ph/0410195.

[16] W. Fulton and J. Harris. Representation Theory, volume 129 of Graduate Texts in Mathematics. Springer, 2004. DOI: 10.1007/978-1-4612-0979-9.

[17] A. Hayashi, T. Hashimoto, and M. Horibe. Reexamination of optimal quantum state estimation of pure states. Physical Review A, 72:032325, 2005. DOI: 10.1103/PhysRevA.72.032325.

[18] P. Hayden, D. Leung, and A. Winter. Aspects of generic entanglement. Communications in Mathematical Physics, 265(1):95-117, 2006. DOI: 10.1007/s00220-006-1535-6.

[19] T. Heinosaari and M. Ziman. The Mathematical Language of Quantum Theory. Cambridge University Press, 2012. DOI: 10.1017/CBO9781139031103.

[20] M. Hillery, V. Bužek, and M. Ziman. Probabilistic implementation of universal quantum processors. Physical Review A, 65(2):022301, 2002. DOI: 10.1103/PhysRevA.65.022301.

[21] M. Hillery, M. Ziman, and V. Bužek. Implementation of quantum maps by programmable quantum processors. Physical Review A, 66(4):042302, 2002. DOI: 10.1103/PhysRevA.66.042302.

[22] M. Hillery, M. Ziman, and V. Bužek. Approximate programmable quantum processors. Physical Review A, 73(2):022345, 2006. DOI: 10.1103/PhysRevA.73.022345.

[23] M. Horodecki, P. Horodecki, and R. Horodecki. General teleportation channel, singlet fraction, and quasidistillation. Physical Review A, 60:1888-1898, 1999. DOI: 10.1103/PhysRevA.60.1888.

[24] S. Ishizaka and T. Hiroshima. Asymptotic teleportation scheme as a universal programmable quantum processor. Physical Review Letters, 101(24):240501, 2008. DOI: 10.1103/physrevlett.101.240501.

[25] A. Jamiołkowski. Linear transformations which preserve trace and positive semidefiniteness of operators. Reports on Mathematical Physics, 3(4):275-278, 1972. DOI: 10.1016/0034-4877(72)90011-0.

[26] M. Keyl. Fundamentals of quantum information theory. Physics Reports, 369(5): 431-548, 2002. DOI: 10.1016/S0370-1573(02)00266-1.

[27] A. M. Kubicki, C. Palazuelos, and D. Pérez-García. Resource quantification for the no-programing theorem. Physical Review Letters, 122(8):080505, 2019. DOI: 10.1103/PhysRevLett.122.080505.

[28] M. Ledoux and M. Talagrand. Probability in Banach Spaces: Isoperimetry and Processes, volume 23 of A Series of Modern Surveys in Mathematics Series. Springer, 1991. DOI: $10.1007 / 978-3-642-20212-4$.

[29] I. Marvian and R. W. Spekkens. Extending Noether's theorem by quantifying the asymmetry of quantum states. Nature Communications, 5:3821, 2014. DOI: $10.1038 /$ ncomms4821. 
[30] M. Mozrzymas, M. Studziński, and N. Datta. Structure of irreducibly covariant quantum channels for finite groups. Journal of Mathematical Physics, 58(5):052204, 2017. DOI: $10.1063 / 1.4983710$.

[31] A. Müller-Hermes. Transposition in quantum information theory. Master's thesis, Technical University of Munich, 2012. URL https://www-m5.ma.tum.de/foswiki/ pub/M5/CQC/mth.pdf.

[32] M. A. Nielsen and I. L. Chuang. Programmable quantum gate arrays. Physical Review Letters, 79(2):321-324, 1997. DOI: 10.1103/PhysRevLett.79.321.

[33] E. Noether. Invariante Variationsprobleme. Nachrichten von der Gesellschaft der Wissenschaften zu Göttingen, Mathematisch-Physikalische Klasse, 1918:235-257, 1918. URL https://de.wikisource.org/wiki/Invariante_Variationsprobleme.

[34] D. Petz. Quantum Information Theory and Quantum Statistics. Theoretical and Mathematical Physics. Springer, 2008. DOI: 10.1007/978-3-540-74636-2.

[35] S. Pirandola, R. Laurenza, C. Ottaviani, and L. Banchi. Fundamental limits of repeaterless quantum communications. Nature Communications, 8(1):15043, 2017. DOI: 10.1038 /ncomms15043.

[36] S. Pirandola, R. Laurenza, C. Lupo, and J. L. Pereira. Fundamental limits to quantum channel discrimination. npj Quantum Information, 5:50, 2019. DOI: 10.1038/s41534019-0162-y.

[37] D. Pérez-García. Optimality of programmable quantum measurements. Physical Review A, 73(5):052315, 2006. DOI: 10.1103/physreva.73.052315.

[38] B. Simon. Representations of Finite and Compact Groups, volume 10 of Graduate Studies in Mathematics. American Mathematical Society, 1996. DOI: $10.1090 / \mathrm{gsm} / 010$.

[39] G. Vidal, L. Masanes, and J. I. Cirac. Storing quantum dynamics in quantum states: A stochastic programmable gate. Physical Review Letters, 88(4):047905, 2002. DOI: 10.1103/PhysRevLett.88.047905.

[40] K. G. H. Vollbrecht and R. F. Werner. Entanglement measures under symmetry. Physical Review A, 64(6):062307, 2001. DOI: 10.1103/PhysRevA.64.062307.

[41] D.-S. Wang. Choi states, symmetry-based quantum gate teleportation, and storedprogram quantum computing. Physical Review A, 101(5):052311, 2020. DOI: 10.1103/PhysRevA.101.052311.

[42] J. Watrous. The Theory of Quantum Information. Cambridge University Press, Cambridge, 2018. DOI: 10.1017/9781316848142.

[43] R. F. Werner and A. S. Holevo. Counterexample to an additivity conjecture for output purity of quantum channels. ournal of Mathematical Physics, 43(9):4353-4357, 2002. DOI: $10.1063 / 1.1498491$.

[44] M. M. Wilde. Quantum Information Theory. Cambridge University Press, 2nd edition, 2017. DOI: $10.1017 / 9781316809976.001$.

[45] M. M. Wilde, M. Tomamichel, and M. Berta. Converse bounds for private communication over quantum channels. IEEE Transactions on Information Theory, 63(3): 1792-1817, 2017. DOI: 10.1109/tit.2017.2648825.

[46] A. Winter. Tight uniform continuity bounds for quantum entropies: Conditional entropy, relative entropy distance and energy constraints. Communications in Mathematical Physics, 347(1):291-313, 2016. DOI: 10.1007/s00220-016-2609-8.

[47] Y. Yang, R. Renner, and G. Chiribella. Optimal universal programming of unitary gates. Physical Review Letters, 125:210501, 2020. DOI: 10.1103/PhysRevLett.125.210501. 